Pacific Journal of Mathematic 


\title{
ON POINT-FREE PARALLELISM AND WILCOX LATTICES
}

\author{
SHÛICHIRô MAEDA
}

\begin{abstract}
A Wilcox lattice $L$ is constructed from a complemented modular lattice $A$, by deleting nonzero elements of some ideal of $A$ and by introducing in the remains $L$ the same order as $\Lambda$. The lattice $A$ is called the modular extension of $L$. Using the theory of parallelism in atomistic lattices, it was proved that any affine matroid lattice is an atomistic Wilcox lattice, that is, an existence theorem of the modular extension in the atomistic case. The main purpose of this paper is to extend this result to the general case, by the use of arguments on point-free parallelism.
\end{abstract}

A matroid lattice is an upper continuous atmistic lattice with the covering property. In the book [2] of Dubreil-Jacotin, Leisieur and Croisot, a generalized affine geometry is defined as a weakly modular matroid lattice of length $\geqq 4$, satisfying the Euclid's weak parallel axiom. This lattice is called an affine matroid lattice in [4] and [5]. In [2], pp. 311-314, it is proved that any affine matroid lattice has the modular extension and hence it is a Wilcox lattice. One can see that the key theorems in the proof of this result are the transitivity theorem of parallelism and theorems on the incomplete elements.

In this paper, we consider a sectionally semicomplemented lattice $L$ with some join-dense set of modular elements (see $\S 1$ ). This is a generalization of an atomistic lattice. Instead of the parallelism in matroid lattices, we use the point-free parallelism introduced by $\mathrm{F}$. Maeda [6]. In $\S 2$, we give some fundamental results on point-free parallelism. In $\S 3$, we introduce three axioms ( $\mathrm{P} 1),(\mathrm{P} 2)$ and $(\mathrm{P} 3)$ on point-free parallelism in $L$, which are satisfied if $L$ is a Wilcox lattice. In the subsequent three sections, we assume that $L$ is weakly modular, left complemented and of length $\geqq 4$, and that $L$ satisfies ( $\mathrm{P}$ 1) and ( $\mathrm{P} 2$ ). The main result in $\S 4$ is the transitivity of pointfree parallelism. In $\S 5$, we define the parallel images of incomplete elements which generalize those defined in [5], $\S 4$. In $\S 6$, adding the axiom ( $\mathrm{P} 3$ ) in a special case, we construct the modular extension of $L$ and we get two main theorems 6.1 and 6.2.

1. Preliminaries. In a lattice $L$, we write $(a, b) M$ when

$$
(c \vee a) \wedge b=c \vee(a \wedge b) \text { for } c \leqq b \text {. }
$$

An element $a \in L$ is called a modular element when $(x, a) M$ for every 
$x \in L$. The elements 0,1 , if they exist, are modular elements. We denote by $\mathscr{C}$ the set of modular elements of $L$ except 0 and 1 . An element $a \in L$ is called a strongly modular element when every element $a_{1}$ with $a_{1} \leqq a$ is modular. We denote by $\mathscr{C}_{s}$ the set of strongly modular elements except 0 and 1.

A lattice $L$ is called $M$-symmetric when $(a, b) M$ implies $(b, a) M$ in $L$. In an $M$-symmetric lattice $L$ with 0 , we write $a \perp b$ when $a \wedge b=0$ and $(a, b) M$. The following properties are easily verified (see [9]): (1) $a \perp a$ implies $a=0$, (2) $a \perp b$ implies $b \perp a$, (3) $a \perp b, a_{1} \leqq a$ imply $a_{1} \perp b$, (4) $a \perp b, a \vee b \perp c$ imply $a \perp b \vee c$. If $a \perp b$ and $a \vee b \perp c$ then we have $a \perp b \vee c$ and $b \perp a \vee c$ by (4), and then we write $(\alpha, b, c) \perp$.

A lattice $L$ with 0 is called left complemented when for every $a, b \in L$ there exists $b_{1} \in L$ such that

$$
b_{1} \leqq b, a \vee b=a \vee b_{1}, a \wedge b_{1}=0 \quad \text { and } \quad\left(b_{1}, a\right) M .
$$

By [10], Theorem 2, any left complemented lattice $L$ is $M$-symmetric, and hence if $a \leqq b$ in $L$ then there exists $c \in L$ such that $a \vee c=b$ and $a \perp c$. Moreover it is easy to show that $L$ is relatively complemented.

A lattice with 0 is called weakly modular when $a \wedge b \neq 0$ implies $(a, b) M$.

A subset $S$ of a lattice $L$ is called join-dense when every $a \in L$ is the join of some elements in $S$. We write $a<b$ when $a<b$ and there is no element $c$ such that $a<c<b$. In a lattice $L$ with 0 , an element $p$ is called an atom when $0 \prec p$. Evidently any atom is a strongly modular element. The set of atoms of $L$ is denoted by $\Omega$. A lattice $L$ with 0 is called atomistic when $\Omega$ is join-dense. We say that $L$ has the covering property when $p \not a, p \in \Omega$ imply $a \prec a \vee p$. It is easily seen that this property is equivalent to $(p, a) M$ for every $p \in \Omega$ and $a \in L$. Hence any $M$-symmetric lattice with 0 has the covering property.

A lattice $L$ with 0 is called semicomplemented when for every $a \in L$ (with $a \neq 1$ if $L$ has 1 ) there exists $c \in L$ such that $c \neq 0$ and $c \wedge a=0 . L$ is called sectionally semicomplemented (in symbols, an $S S C$ lattice) when every interval $L[0, b]$ is semicomplemented, that is, for $a, b \in L$ with $a<b$ there exists $c \in L$ such that $0 \neq c \leqq b$ and $c \wedge a=0$. Let $\mathscr{C}$ be a set of nonzero elements of $L . L$ is called $\mathscr{C}$-SSC when for $a, b \in L$ with $a<b$ there exists $c \in \mathscr{C}$ such that $c \leqq b$ and $c \wedge a=0$. The following statements are easily verified: (1) If $p$ is an atom of a $\mathscr{C}-S S C$ lattice then $p$ must be in $\mathscr{C},(2)$ $L$ is atomistic if and only if $L$ is $Q-S S C$, (3) $L$ is $\mathscr{C}-S S C$ if and only if $L$ is $S S C$ and $\mathscr{C}$ is join-dense in $L$. 
The length of a lattice $L$ is the least upper bound of the lengths of the chains in $L$ (see [1], p. 5).

Lemma 1.1. ${ }^{1}$ Let $a<b$ in an $M$-symmetric lattice $L$. If there exists a connected chain $a=a_{0} \prec a_{1} \prec \cdots \prec a_{n}=b$ of length $n$, then all chains from a to $b$ have length $\leqq n$.

Proof. If $n=1$, the lemma is trivial. Suppose this is true for $n-1$. Let $a=a_{0} \prec a_{1} \prec \cdots \prec a_{n}=b$ and $a=b_{0}<b_{1}<\cdots<b_{m}=b$, and we shall show $m \leqq n$. When $a_{1} \leqq b_{1}$, we have $a_{1}<b_{2}<\cdots<b_{m}=$ $b$. Hence $m-1 \leqq n-1$ by the induction hypothesis. When $a_{1} \not b_{1}$, let $r(<m)$ be greatest such that $a_{1} \not b_{r}$. For $i=1, \cdots, r$, we have $a_{1} \wedge b_{i}=a$ and $\left(b_{i}, a_{1}\right) M$, since $a_{1}$ covers $a$. Then $\left(a_{1}, b_{i}\right) M$ by $M$-symmetry. If $a_{1} \vee b_{i-1}=a_{1} \vee b_{i}$, then

$$
b_{i}=\left(a_{1} \vee b_{i}\right) \wedge b_{i}=\left(b_{i-1} \vee a_{1}\right) \wedge b_{i}=b_{i-1} \vee\left(a_{1} \wedge b_{i}\right)=b_{i-1},
$$

a contradiction. Hence we have

$$
a_{1}=a_{1} \vee b_{0}<a_{1} \vee b_{1}<\cdots<a_{1} \vee b_{r} \leqq b_{r+1}<b_{r+2}<\cdots<b_{m}=b .
$$

Hence, by the induction hypothesis, we get $m-1 \leqq n-1$. Therefore the lemma is true for $n$.

It follows from this lemma that if the length of $L[a, b]>n$ then there is no connected chain of length $n$ from $a$ to $b$.

2. Point-free parallelism. Let $L$ be a lattice with 0 , and let $a$ and $b$ be nonzero elements of $L$. If $a \wedge b=0$ and there exists $m \in \mathbb{l}$ such that $m \leqq a$ and $m \vee b=a \vee b$ then we write $a<l_{(m)} b$. If $a<\left.\right|_{(m)} b$ and $b<\left.\right|_{(n)} a$ then we write $a \|_{(m, n)} b$ and we say that $a$ and $b$ are parallel with axes $m$ and $n$. We remark that $a \|_{(m, n)} b$ if and only if $a \wedge b=0$ and there exist $m, n \in \mathscr{C}$ such that $m \leqq a$, $n \leqq b$ and $m \vee b=a \vee n$.

Lemma 2.1. If $a<\left.\right|_{(m)} b$ then $m$ is maximal in the set $\{n \in \mathscr{M}$ : $n \leqq a\}$.

Proof. Let $n$ be an element of $\mathscr{C}$ with $m \leqq n \leqq a$. Then since $n$ is modular and $n \wedge b \leqq a \wedge b=0$, we have

$$
n=(a \vee b) \wedge n=(m \vee b) \wedge n=m \vee(b \wedge n)=m \text {. }
$$

LEMMA 2.2. Let $a<\left.\right|_{(m)} b$.

(i) If $m \leqq a_{1}<a$ then $a_{1}<l_{(m)} b$.

1 The author is indebted to the referee for this lemma. 
(ii) If $b<b_{1}$ and $a \wedge b_{1}=0$ then $a<\left.\right|_{(m)} b_{1}$.

(iii) If $b<b_{1}$ and $m<b_{1}$ then $a<b_{1}$.

These statements can easily be proved.

Lemma 2.3. In an M-symmetric lattice with 0 , if $a \|_{(m, n)} b$ and $a=m$ then $b=n$.

Proof. Since $L$ is $M$-symmetric, we have $(m, b) M$. Since $b \leqq$ $a \vee n=m \vee n$ and $m \wedge b=0$, we have

$$
b=(n \vee m) \wedge b=n \vee(m \wedge b)=n .
$$

Lemma 2.4. In an $M$-symmetric lattice with 0 ,

(i) if $a \wedge b=0$ and $a \vee b \perp c$ then $a \wedge(b \vee c)=0$,

(ii) if $a<\left.\right|_{(m)} b$ and $a \vee b \perp c$ then $a<\left.\right|_{(m)} b \vee c$.

Proof. (i ) Since $c \perp a \vee b$, we have $(b \vee c) \wedge(a \vee b)=b \vee$ $\{c \wedge(a \vee b)\}=b$. Hence $a \wedge(b \vee c)=a \wedge b=0$.

(ii) We have $a \wedge(b \vee c)=0$ by (i). Moreover, $m \vee b \vee c=$ $a \vee b \vee c$ by $a<\left.\right|_{(m)} b$. Hence $a<\left.\right|_{(m)} b \vee c$.

LeMma 2.5. In a weakly modular lattice,

(i) if $a \wedge b=0, m \leqq a, n \leqq b$ where $m, n \in \mathscr{l l}$ then $a \wedge(m \vee$ b) $\|_{(m, n)} b \wedge(n \vee a)$,

(ii) if $a<\left.\right|_{(m)} b$ and $n \leqq b$ where $n \in \mathbb{C l}$ then $a \|_{(m, n)} b \wedge(n \vee a)$.

Proof. ( i ) We have $a \wedge(m \vee b) \wedge b \wedge(n \vee a)=a \wedge b=0$. Since $a \wedge(m \vee b) \geqq m>0$, we have $(a, m \vee b) M$ and similarly $(b$, $n \vee a) M$. Hence

$$
n \vee\{a \wedge(m \vee b)\}=(n \vee a) \wedge(m \vee b)=m \vee\{b \wedge(n \vee a)\} .
$$

Thus, $a \wedge(m \vee b) \|_{(m, n)} b \wedge(n \vee a)$. (ii) follows from (i) evidently.

LEMMA 2.6. In a weakly modular, $M$-symmetric lattice $L$, if $a \|_{(m, n)} b$, then there exist mutually inverse, isomorphic mappings between the intervals $L[m, a]$ and $L[n, b]$, which are defined by $a_{1} \rightarrow b \wedge$ $\left(n \vee a_{1}\right)$ for $a_{1} \in L[m, a]$ and $b_{1} \rightarrow a \wedge\left(m \vee b_{1}\right)$ for $b_{1} \in L[n, b]$.

Proof. [6], Theorem 2.12.

Lemma 2.7. Let $a<\left.\right|_{(m)} c$ in a weakly modular, $M$-symmetric, IC-SSC lattice $L$ with 1. If $n \in \mathscr{C l}, n \wedge a=0$ and $n \vee a<1$ then there exists $b \in L$ such that $a \|_{(m, n)} b$. 
Proof. ( i ) When $c \$ n \vee a$, we have $(n \vee a) \wedge c<c$. There exists $n^{\prime} \in \mathscr{l}$ such that $n^{\prime} \leqq c$ and $n^{\prime} \wedge(n \vee a)=0$. Putting $c^{\prime}=$ $c \wedge\left(n^{\prime} \vee a\right)$, we have $a \|_{\left(m, n^{\prime}\right)} c^{\prime}$ by Lemma 2.5 (ii). Since $n \perp a$ and $n \vee a \perp n^{\prime}$, we have $n \perp a \vee n^{\prime}=a \vee c^{\prime}$. Hence, by Lemma 2.4 (ii), we have $a<\left.\right|_{(m)} c^{\prime} \vee n$. Putting $b=\left(c^{\prime} \vee n\right) \wedge(n \vee a)$, we have $a \|_{(m, n)} b$ by Lemma 2.5 (ii).

(ii) When $c \leqq n \vee a(<1)$, we take $n^{\prime} \in \mathscr{l l}$ with $(n \vee a) \wedge n^{\prime}=0$. Then $c \vee a \perp n^{\prime}$, since $(c \vee a) \wedge n^{\prime} \leqq(n \vee a) \wedge n^{\prime}=0$. Putting $c^{\prime}=$ $c \vee n^{\prime}$, we have $a<\left.\right|_{(m)} c^{\prime}$ by Lemma 2.4 (ii). Since $c^{\prime} \notin n \vee a$, by (i) there exists $b$ such that $a \|_{(m, n)} b$.

3. Parallelism in Wilcox lattices. A Wilcox lattice $L$ is constructed in the following manner (see [9] and [6]). Let $\Lambda$ be a given complemented modular lattice whose lattice operations are denoted by $\cup$ and $\cap$. Let $S$ be a fixed proper ideal of $\Lambda$ with 0 deleted ( $S$ may be empty). As ordering of the set $L \equiv \Lambda-S$ we use that of $A$. Then $L$ is a lattice having the following properties, where $a, b \in L$ and we denote the lattice operations in $L$ by $\vee$ and $\wedge$ :

(W 1) $a \vee b=a \cup b$ for all $a, b \in L$,

(W 2) $a \wedge b= \begin{cases}a \cap b & \text { if } a \cap b \in L \\ 0 & \text { if } a \cap b \in S,\end{cases}$

(W 3) $(a, b) M$ in $L$ if and only if $a \cap b \in L$.

By (W 2) and (W 3), $L$ is weakly modular and $M$-symmetric. Moreover, $a \perp b$ in $L$ if and only if $a \cap b=0$.

We call $A$ the modular extension of $L$. An element in $S$ is called an imaginary element for $L$, and when $S$ has a greatest element $i$ then it is called the imaginary unit. A nonzero element $a$ of $L$ is called regular when $a \cap u=0$ for every $u \in S$. The set of regular elements is denoted by $R$. Evidently, $S$ is empty if and only if $1 \in R$.

LEMMA 3.1. Let $L \equiv \Lambda-S$ be a semicomplemented Wilcox lattice, and let $a$ be an element of $L$ with $0<a<1$. The following three statements are equivalent.

$(\alpha)$ a is regular. ( $\beta)$ a is modular. ( $\gamma)$ a is strongly modular.

Proof. $(\alpha) \Longrightarrow(\gamma)$. Let $b \in L$ and $a_{1} \leqq a$ in $L$. If $b \cap a_{1} \in S$, then, since $a$ is regular, we have $0=a \cap\left(b \cap a_{1}\right)=b \cap a_{1}$, a contradiction. Hence $b \cap a_{1} \in L$, and then $\left(b, a_{1}\right) M$ by (W 3). Therefore $a$ is strongly modular. $(\gamma) \Longrightarrow(\beta)$ is trivial. $(\beta) \Longrightarrow(\alpha)$. Since $L$ is semicomplemented, there exists $c \in L$ such that $c \neq 0$ and $c \wedge a=0$. Since $a$ is modular, we have $c \cap a \in L$ by (W 3). Hence $c \cap a=c \wedge a=0$ by (W 2). For an arbitrary $u \in S$, we put $b=c \cup(a \cap u)$. Since $b \in L$, we have $(b, a) M$ and hence $b \cap a \in L$. By the modularity of $\Lambda$, 


$$
b \cap a=\{(a \cap u) \cup c\} \cap a=(a \cap u) \cup(c \cap a)=a \cap u .
$$

Hence $a \cap u \in L$, and then $a \cap u=0$. Therefore $a$ is regular.

Lemma $3.2 .^{2}$ (i) A Wilcox lattice $L \equiv \Lambda-S$ is left complemented if and only if it satisfies the following condition:

(1) If $a<b$ in $L$ then there exists $c \in L$ such that $0 \neq c \leqq b$, $c \wedge a=0$ and $(c, a) M$.

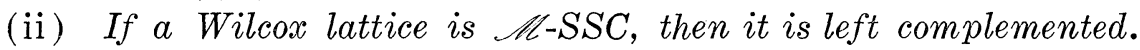

Proof. If $L$ is left complemented then evidently (1) is satisfied. Conversely, assume that (1) is satisfied in $L$. Let $a, b \in L$. We shall show that

(2) There exists $b_{1} \in L$ such that $b=(a \cap b) \cup b_{1}$ and $(a \cap b) \cap$ $b_{1}=0$.

When $a \cap b \in S$, we take a complement $b_{1}$ of $a \cap b$ in the interval $A[0, b]$. Then $b_{1} \in L$, since otherwise $b=b_{1} \cup(a \cap b) \in S$, a contradiction. Hence $b_{1}$ has the desired property. When $a \cap b \in L$, we may assume $a \cap b<b$. By (1) there exists $c \in L$ such that $0 \neq c \leqq b, c \wedge$ $(a \cap b)=0$ and $(c, a \cap b) M$. Then we have $c \cap(a \cap b)=0$. Let $\lambda$ be a complement of $(a \cap b) \cup c$ in $\Lambda[0, b]$, and put $b_{1}=c \cup \lambda$. Since $(a \cap b, c, \lambda) \perp$ in $A, b_{1}$ is a complement of $a \cap b$ in $A[0, b]$. Moreover, since $0 \neq c \in L$, we have $b_{1} \in L$. Thus (2) has been proved.

By (2), we have $a \vee b_{1}=a \cup b_{1}=a \cup b=a \vee b$, and since $a \cap b_{1}=$ $a \cap b \cap b_{1}=0$, we have $a \wedge b_{1}=0$ and $\left(b_{1}, a\right) M$. Therefore $L$ is left complemented. The second statement of the lemma follows from the first one.

REMARK 3.1. Let $L \equiv \Lambda-S$ be a Wilcox lattice with the imaginary unit $i$, and assume that $L$ is $S S C$. For any nonzero element a of $L$, there exists $a_{1} \in \mathscr{l}$ such that $a_{1} \leqq a$; because a complement $a_{1}$ of $a \cap i$ in $\Lambda[0, a]$ is regular since $a_{1} \cap i=0$, and hence $a_{1} \in \mathscr{M l}$ by Lemma 3.1. Therefore $L$ is $\mathscr{L}-S S C$ and then it is left complemented.

Definition. An element $a$ of a Wilcox lattice $L \equiv \Lambda-S$ is called irregular when there exist $m \in R$ and $u \in S$ such that $\alpha=m \cup u$ (see [6]). We call $u$ an imaginary part of $a$, and denote it by $\iota(a)$; while $m$ is called a regular part of $a$. For a regular element a we put $\iota(a)=0$. It is easy to show that if a Wilcox lattice $L$ has the imaginary unit $i$, then every nonzero element of $L$ is either regular or irregular.

LEMMA 3.3. If $a$ is an irregular element of a Wilcox lattice $L \equiv \Lambda-S$, then an imaginary part $c(a)$ is uniquely determined by

${ }^{2}$ This lemma is suggested by the referee. 
$a$, and it is the greatest element of $S$ contained in $a$.

Proof. Let $a=m \cup u, m \in R$ and $u \in S$. If $v \in S$ and $v \leqq a$, then since $u \cup v \in S$, we have $m \cap(u \cap v)=0$. Hence by the modularity of $\Lambda$,

$$
u=u \cup\{m \cap(u \cup v)\}=(u \cup m) \cap(u \cup v)=a \cap(u \cup v) \geqq v .
$$

REMARK 3.2. A regular part of an irregular element $a$ is a complement of $\iota(\alpha)$ in $\Lambda[0, a]$. This is not necessarily unique. It is easy to show by Lemma 3.3 that a Wilcox lattice $L$ has the imaginary unit if and only if the unit 1 of $L$ is irregular.

LEMMA 3.4. Let $a$ and $b$ be nonzero elements of a Wilcox lattice $L \equiv \Lambda-S$. The following statements are equivalent.

( $\alpha) \quad a<\left.\right|_{(m)} b$ with $a \neq m \in R$.

( $\beta) \quad a \cap b \in S$ and $a=m \cup(a \cap b)$ with $m \in R$.

Each of $(\alpha)$ and $(\beta)$ implies that $a$ is irregular and $\iota(a)=a \cap b$.

Proof. [6], Theorem 3.8.

REMARK 3.3. In a Wilcox lattice, if $a<\left.\right|_{(m)} b$ with $m \in R$ then $a=m \cup \iota(\alpha)$ by Lemma 3.4 and by the fact that $a=m$ implies $\iota(\alpha)=0$.

LEMMA 3.5. Let $a$ and $b$ be irregular elements of a Wilcox lattice $L \equiv \Lambda-S$. If $a<\left.\right|_{(m)} b$ with $m \in R$ then $\iota(a) \leqq \iota(b)$, and if $a \|_{(m, n)} b$ with $m, n \in R$ then $\iota(a)=\iota(b)$.

Proof. This lemma is evident by Lemmas 3.3 and 3.4 .

Lemma 3.6. In a Wilcox lattice $L \equiv A-S$, let $a=m \cup u$ and $b=n \cup u$ where $m, n \in R$ and $u \in S$. If either $a \wedge n=0$ or $b \wedge m=0$ then $a \|_{(m, n)} b$.

Proof. [6], Lemma 3.10.

Parallel Axioms. Let $\mathscr{C}$ be a subset of $\mathscr{C}$. We consider the following three parallel axioms with respect to $\mathscr{C}$.

(P 1) If $a \|_{\left(m_{1}, n\right)} b_{1}$ and $a \|_{\left(m_{2} \cdot n\right)} b_{2}$ where $m_{1}, m_{2}, n \in \mathscr{C}$, then $b_{1}=b_{2}$.

(P 2) If $a_{1} \|_{(m, n)} b_{1}$ and $a_{2} \|_{(m, n)} b_{2}$ where $m, n \in \mathscr{C}$ and if $\left(a_{1} \vee a_{2}\right) \wedge$ $n=0$, then $a_{1} \vee a_{2} \|_{(m, n)} b_{1} \vee b_{2}$.

(P 3) If $a_{1}<\left.\right|_{(m)} b_{1}$ and $a_{2}<\left.\right|_{(m)} b_{2}$ where $m \in \mathscr{C}$ and if $a_{1} \vee a_{2}=1$, then for any $a$ with $m<a<1$ there exists $b$ such that $a<\left.\right|_{(m)} b$.

Lemma 3.7. If $L \equiv \Lambda-S$ is a semicomplemented Wilcox lattice 
then ( $\mathrm{P}$ 1), ( $\mathrm{P}$ 2) and (P 3) are satisfied for any subsets $\mathscr{C}$ of $\mathscr{K}$.

Proof. (i) We have $\mathscr{C} \subset R$ by Lemma 3.1. Let $a \|_{\left(m_{1}, m\right)} b_{1}$ and $a \|_{\left(m_{2}, n\right)} b_{2}$ where $m_{1}, m_{2}, n \in \mathscr{C} \subset R$. It follows from Remark 3.3 and Lemma 3.5 that $b_{i}=n \cup \iota\left(b_{i}\right)(i=1,2)$ and $\iota\left(b_{1}\right)=\iota(a)=\iota\left(b_{2}\right)$. Hence $b_{1}=b_{2}$.

(ii) Let $a_{1} \|_{(m, n)} b_{1}$ and $a_{2} \|_{(m, n)} b_{2}$ where $m, n \in \mathscr{C}$, and assume that $\left(a_{1} \vee a_{2}\right) \wedge n=0$. We have $a_{i}=m \cup \iota\left(a_{i}\right), \quad b_{i}=n \cup \iota\left(b_{i}\right)$ and $\iota\left(a_{i}\right)=\iota\left(b_{i}\right)(i=1,2)$. We put $u=\iota\left(a_{1}\right) \cup \iota\left(a_{2}\right)=\iota\left(b_{1}\right) \cup \iota\left(b_{2}\right)$. Then we have $u \in S, \quad a_{1} \vee a_{2}=a_{1} \cup a_{2}=m \cup u$ and $b_{1} \vee b_{2}=b_{1} \cup b_{2}=n \cup u$. Hence $a_{1} \vee a_{2} \|_{(m, n)} b_{1} \vee b_{2}$ by Lemma 3.6.

(iii) Let $a_{1}<\left.\right|_{(m)} b_{1}$ and $a_{2}<\left.\right|_{(m)} b_{2}$ where $m \in \mathscr{C}$, and assume that $a_{1} \vee a_{2}=1$. Since $a_{i}=m \cup \iota\left(a_{i}\right)$, putting $u=\iota\left(a_{1}\right) \cup \iota\left(a_{2}\right)$, we have $u \in S$ and $m \cup u=a_{1} \cup a_{2}=a_{1} \vee a_{2}=1$. If $m<a<1$, then we have $m \cup(u \cap a)=(m \cup u) \cap a=a$, and moreover $u \cap a \in S$, since otherwise $u \cap a=0$ and then $m=a$. Since $L$ is semicomplemented, there exists $c \in L$ with $c \neq 0$ and $c \wedge a=0$. Then $c \cap a \in S \smile\{0\}$. Putting $b=$ $c \cup(u \cap a)$, we have

$$
a \cap b=\{(u \cap a) \cup c\} \cap a=(u \cap a) \cup(c \cap a) \in S .
$$

Moreover,

$$
a=m \cup(u \cap a)=m \cup(u \cap a) \cup(c \cap a)=m \cup(a \cap b) .
$$

Hence $a<l_{(m)} b$ by Lemma 3.4.

4. Transitivity of parallelism. We consider the following condition for a subset $\mathscr{C}$ of $\mathscr{C}$ :

(C 1) If $m \in \mathscr{C}$ and $0<m_{1}<m$ then $m_{1} \in \mathscr{C}$.

For instance, in a lattice with 0 , both the set $\Omega$ of atoms and the set $\mathscr{C}_{s}$ of strongly modular elements satisfy (C 1), and also the set of regular elements of a Wilcox lattice does. Evidently, if $\mathscr{C}$ satisfies (C 1) then $\mathscr{C} \subset \mathscr{H}_{s}$.

In this and the next sections, let $L$ be a weakly modular, left complemented lattice, with 0 and 1 , of length $\geqq 4$ (may be infinite), and assume that for some fixed subset $\mathscr{C}$ of $\mathscr{C}$, satisfying (C 1$), L$ is $\mathscr{C}-S S C$ and $L$ satisfies the axioms (P 1$)$ and (P 2) with respect to $\mathscr{C}$.

For any $m \in \mathscr{C}, L[m, 1]$ is a modular lattice, since $L$ is weakly modular. We put $\mathscr{C}^{\prime}=\{m \in \mathscr{C}$; the length of $L[m, 1] \geqq 3\}$. Evidently, $\mathscr{C}^{\prime}$ satisfies (C 1 ). Since the length of $L \geqq 4$, it is easily seen that for any $m \in \mathscr{C}$ there exists $m_{1} \in \mathscr{C}^{\prime}$ such that $m_{1} \leqq m$. Hence $L$ is $\mathscr{C}^{\prime}-S S C$. Therefore, taking $\mathscr{C}^{\prime}$ instead of $\mathscr{C}$, we may assume that

(C 2) the length of $L[m, 1] \geqq 3$ for every $m \in \mathscr{C}$. 
LEMMA 4.1. In $L$, if $a \|_{\left(m_{1}, n\right)} b_{1}, a<\left.\right|_{\left(m_{2}\right)} b_{2}$ and $n \leqq b_{2}$ where $m_{1}$, $m_{2}, n \in \mathscr{C}$ then $b_{1} \leqq b_{2}$.

Proof. We have $a \|_{\left(m_{2}, n\right)} b_{2} \wedge(n \vee a)$ by Lemma 2.5 (ii). Hence by $(\mathrm{P} 1), b_{1}=b_{2} \wedge(n \vee a) \leqq b_{2}$.

LeMmA 4.2. In $L$, if $a\left\|_{\left(m_{1}, n_{1}\right)} b_{1}, a\right\|_{\left(m_{2}, n_{2}\right)} b_{2}$ where $m_{1}, m_{2}, n_{1}, n_{2} \in \mathscr{C}$ and $\left(a, n_{1}, n_{2}\right) \perp$ then $b_{1} \|_{\left(n_{1}, n_{2}\right)} b_{2}$ and $a \wedge\left(b_{1} \vee b_{2}\right)=0$.

Proof. Since $n_{1} \perp a \vee n_{2}$, we have $b_{1} \wedge b_{2} \leqq\left(a \vee n_{1}\right) \wedge\left(a \vee n_{2}\right)=$ $a \vee\left\{n_{1} \wedge\left(a \vee n_{2}\right)\right\}=a$. Hence $b_{1} \wedge b_{2}=a \wedge b_{1} \wedge b_{2}=0$. Next, since $a \vee b_{1}=a \vee n_{1}$, we have $a \vee b_{1} \perp n_{2}$. By Lemma 2.4 (ii), we have $a<\left.\right|_{\left(m_{1}\right)} b_{1} \vee n_{2}$. Hence $b_{2} \leqq b_{1} \vee n_{2}$ by Lemma 4.1. Similarly $b_{1} \leqq$ $b_{2} \vee n_{1}$. Therefore, $b_{1} \vee n_{2}=b_{2} \vee n_{1}$, and hence $b_{1} \|_{\left(n_{1}, n_{2}\right)} b_{2}$. Moreover, $a \wedge\left(b_{1} \vee b_{2}\right)=a \wedge\left(b \vee n_{2}\right)=0$.

Definition. Let $m \in \mathscr{C}$. An element $a$ of $L$ is called an incomplete element over $m$ when there exists $b \in L$ such that $a<l_{(m)} b$. The set of all incomplete elements over $m$ is denoted by $I_{m}$. It follows from Lemma 4.2 (i) that if $a \in I_{m}$ and $m \leqq a_{1} \leqq a$ then $a_{1} \in I_{m}$. The following theorem shows a fundamental property of incomplete elements.

THEOREM 4.1. If $a \in I_{m}$ and $n \wedge a=0$ where $m, n \in \mathscr{C}$, then there exists a unique element $b$ such that $a \|_{(m, n)} b$.

Proof. The uniqueness follows from (P 1). We shall show the existence. When $a=m$, we may take $b=n$. When $n \vee a<1$, the existence of $b$ follows from Lemma 2.7. Now we assume that $m<a$ and $n \vee a=1$.

When $n$ is not an atom, there exist $n_{1}, n_{2} \in \mathscr{C}$ such that $n=$ $n_{1} \vee n_{2}$ and $n_{1} \perp n_{2}$, since $L$ is left complemented and $\mathscr{C}$ satisfies (C 1). Since $\left(a, n_{1}, n_{2}\right) \perp$, we have $n_{i} \vee a<1(i=1,2)$, and hence there exist $b_{1}, b_{2}$ such that $a \|_{\left(m, n_{i}\right)} b_{i}$ by Lemma 2.7. By Lemma 4.2 we have $a \wedge\left(b_{1} \vee b_{2}\right)=0$. Moreover, $a \vee n=a \vee n_{1} \vee n_{2}=m \vee b_{1} \vee b_{2}$. Hence we have $a \|_{(m, n)} b_{1} \vee b_{2}$.

When $n$ is an atom, we have $a \prec a \vee n=1$ by the covering property. Since $L[m, 1]$ is a modular lattice of length $\geqq 3$ by (C 2 ), there exists $a_{1}$ such that $m<a_{1}<a$. Since $L$ is left complemented, there exist nonzero elements $c_{1}$ and $c_{2}$ such that $m \vee c_{1}=a_{1}, m \perp c_{1}$, $a_{1} \vee c_{2}=a$ and $a_{1} \perp c_{2}$. Since $\left(a_{1}, c_{2}, n\right) \perp$, we have $n \vee a_{1}<1$. Putting $a_{2}=m \vee c_{2}$, since $\left(a_{2}, c_{1}, n\right) \perp$, we have $n \vee a_{2}<1$. Since $a_{1}$, $a_{2} \in I_{m}$, there exist $b_{1}, b_{2}$ such that $a_{i} \|_{(m, n)} b_{i}$. Then by (P 2) we have $a \|_{(m, n)} b_{1} \vee b_{2}$. 
COROLLARY. If $a_{1}, a_{2} \in I_{m}$ where $m \in \mathscr{C}$ and if $a_{1} \vee a_{2}<1$, then $a_{1} \vee a_{2} \in I_{m}$.

Proof. Since $L$ is $\mathscr{C}-S S C$, there exists $n \in \mathscr{C}$ such that $\left(a_{1} \vee a_{2}\right) \wedge$ $n=0$. By Thorem 4.1, there exist $b_{1}$ and $b_{2}$ such that $a_{i} \|_{(n, n)} b_{i}(i=$ 1,2). Hence $a_{1} \vee a_{2} \|_{(m, n)} b_{1} \vee b_{2}$ by (P 2).

LEMMA 4.3. If $a \|_{\left(m, n_{1}\right)} b_{1}$ and $a \|_{\left(m, n_{2}\right)} b_{2}$ where $m, n_{1}, n_{2} \in \mathscr{C}$ and $n=n_{1} \vee n_{2} \in \mathscr{C}$ and if $a \wedge n=0$, then $a \|_{(m, n)} b_{1} \vee b_{2}$.

Proof. It follows from Theorem 4.1 that there exists $b$ such that $a \|_{(m, n)} b$. Since $a \|_{\left(m, n_{1}\right)} b_{1}, a<\left.\right|_{(m)} b$ and $n_{1} \leqq n \leqq b$, we have $b_{1} \leqq b$ by Lemma 4.1. Similarly $b_{2} \leqq b$. Hence $a \wedge\left(b_{1} \vee b_{2}\right) \leqq a \wedge b=0$. Moreover $a \vee n=a \vee n_{1} \vee n_{2}=m \vee b_{1} \vee b_{2}$. Hence $a \|_{(m, n)} b_{1} \vee b_{2}$.

Lemma 4.4. If $a \|_{\left(m, n_{1}\right)} b_{1}$ and $a \|_{\left(m, n_{2}\right)} b_{2}$ where $m, n_{1}, n_{2} \in \mathscr{C}$ and if $b_{1} \wedge n_{2}=0$ then $b_{2} \wedge n_{1}=0$.

Proof. If $b_{2} \wedge n_{1} \neq 0$, then putting $n_{1}^{\prime}=b_{2} \wedge n_{1}$, we have $n_{1}^{\prime} \in \mathscr{C}$, $n_{1}^{\prime} \leqq b_{1}$ and $n_{1}^{\prime} \leqq b_{2}$. Since $a<\left.\right|_{(m)} b_{i}(i=1,2)$, we have $a \|_{\left(m, n_{1}^{\prime}\right)} b_{i} \wedge$ $\left(n_{1}^{\prime} \vee a\right)$ by Lemma 2.5 (ii). Hence $b_{1} \wedge\left(n_{1}^{\prime} \vee a\right)=b_{2} \wedge\left(n_{1}^{\prime} \vee a\right)$ by (P 1), and hence

$$
n_{2} \wedge\left(n_{1}^{\prime} \vee a\right)=n_{2} \wedge b_{2} \wedge\left(n_{1}^{\prime} \vee a\right)=n_{2} \wedge b_{1} \wedge\left(n_{1}^{\prime} \vee a\right)=0 .
$$

Thus we have $\left(a, n_{1}^{\prime}, n_{2}\right) \perp$. It follows from Lemma 4.2 that $b_{2} \wedge$ $\left(n_{1}^{\prime} \vee a\right) \|_{\left(n_{1}^{\prime}, n_{2}\right)} b_{2}$, which is a contradiction since $0<b_{2} \wedge\left(n_{1}^{\prime} \vee a\right) \leqq b_{2}$.

THEOREM 4.2. (Transitivity of parallelism) If $a \|_{\left(m_{1}, n_{1}\right)} b_{1}$ and $a \|_{\left(m_{2}, n_{2}\right)} b_{2}$ where $m_{1}, m_{2}, n_{1}, n_{2} \in \mathscr{C}$ and if $b_{1} \wedge n_{2}=0$, then $b_{1} \|_{\left(n_{1}, n_{2}\right)} b_{2}$.

Proof. (i ) When $\left(a \vee b_{1}\right) \wedge n_{2}=0$, we have $\left(a, n_{1}, n_{2}\right) \perp$. Hence it follows from Lemma 4.2 that $b_{1} \|_{\left(n_{1}, n_{2}\right)} b_{2}$.

(ii) When $n_{2} \leqq a \vee b_{1}<1$, there exists $n \in \mathscr{C}$ such that $a \vee b_{1} \perp n$. Since $n \wedge a=0$, it follows from Theorem 4.1 that there exists $b$ such that $a \|_{\left(m_{1}, n\right)} b$. Since $\left(a \vee b_{1}\right) \wedge n=0$, we have $b_{1} \|_{\left(n_{1}, n\right)} b$ by (i). On the other hand, since $a \vee b_{2}=a \vee n_{2} \leqq a \vee b_{1}$, we have $\left(a \vee b_{2}\right) \wedge n=0$. Hence $b_{2} \|_{\left(n_{2}, n\right)} b$ by (i). Now, we have $\left(n_{2}, b_{1}, n\right) \perp$, since $n_{2} \vee b_{1} \leqq$ $a \vee b_{1} \perp n$. Since $b \vee n_{1}=b_{1} \vee n$, we have $\left(b, n_{1}, n_{2}\right) \perp$. Hence, $b_{1} \|_{\left(n_{1}, n_{2}\right)} b_{2}$ by Lemma 4.2 .

(iii) When $a \vee b_{1}<1$, we put $n_{1}^{\prime}=\left(a \vee b_{1}\right) \wedge n_{2}$. Since $L$ is left complemented, there exists $n_{2}^{\prime \prime}$ such that $n_{2}=n_{2}^{\prime} \vee n_{2}^{\prime \prime}$ and $n_{2}^{\prime} \perp n_{2}^{\prime \prime}$. If $n_{2}^{\prime}=0$ or $n_{2}^{\prime \prime}=0$ then we have $b_{1} \|_{\left(n_{1}, n_{2}\right)} b_{2}$ by (i) or (ii). Let $n_{2}^{\prime} \neq 0$ and $n_{2}^{\prime \prime} \neq 0$. Then $n_{2}^{\prime}, n_{2}^{\prime \prime} \in \mathscr{C}$, and by Theorem 4.1 there exist $b_{2}^{\prime}$ and $b_{2}^{\prime \prime}$ such that $a \|_{\left(m_{2}, n_{2}^{\prime}\right)} b_{2}^{\prime}$ and $a \|_{\left(m_{2}, n_{2}^{\prime \prime}\right)} b_{2}^{\prime \prime}$. Since $a \|_{\left(m_{2}, n_{2}\right)} b_{2}^{\prime} \vee b_{2}^{\prime \prime}$ by 
Lemma 4.3 , we have $b_{2}=b_{2}^{\prime} \vee b_{2}^{\prime \prime}$ by (P 1). Since $n_{2}^{\prime} \leqq \alpha \vee b_{1}$, we have $b_{1} \|_{\left(n_{1}, n_{2}^{\prime}\right)} b_{2}^{\prime}$ by (ii), and since $n_{2}^{\prime \prime} \wedge\left(a \vee b_{1}\right)=n_{2}^{\prime \prime} \wedge\left(a \vee b_{1}\right) \wedge n_{2}=$ $n_{2}^{\prime \prime} \wedge n_{2}^{\prime}=0$, we have $b_{1} \|_{\left(n_{1}, n_{2}^{\prime \prime}\right)} b_{2}^{\prime \prime}$ by (i). By Lemma 4.3 again, we get $b_{1} \|_{\left(n_{1}, n_{2}\right)} b_{2}^{\prime} \vee b_{2}^{\prime \prime}=b_{2}$.

(iv) Let $a \vee b_{1}=1$. When $n_{1}$ is not an atom, there exist $n_{1}^{\prime}$, $n_{1}^{\prime \prime} \in \mathscr{C}$ such that $n_{1}=n_{1}^{\prime} \vee n_{1}^{\prime \prime}$ and $n_{1}^{\prime} \perp n_{1}^{\prime \prime}$. As above there exist $b_{1}^{\prime}$ and $b_{1}^{\prime \prime}$ such that $a\left\|_{\left(m_{1}, n_{1}^{\prime}\right)} b_{1}^{\prime}, a\right\|_{\left(m_{1}, n_{1}^{\prime \prime}\right)} b_{1}^{\prime \prime}$ and $b_{1}=b_{1}^{\prime} \vee b_{1}^{\prime \prime}$. Since $b_{1}^{\prime} \wedge n_{2}=0$ and $a \vee b_{1}^{\prime}=a \vee n_{1}^{\prime}<1$, we have $b_{1}^{\prime} \|_{\left(n_{1}^{\prime}, n_{2}\right)} b_{2}$. Similarly, $b_{1}^{\prime \prime} \|_{\left(n_{1}^{\prime}, n_{2}\right)} b_{2}$. Since $b_{2} \wedge n_{1}=0$ by Lemma 4.4, we have $b_{1} \|_{\left(n_{1}, n_{2}\right)} b_{2}$ by Lemma 4.3. When $n_{1}$ is an atom, we have $a \prec a \vee n_{1}=1$. Then, by the same way as in the proof of Theorem 4.1, there exist elements $a^{\prime}$ and $a^{\prime \prime}$ such that $m_{1} \leqq a^{\prime} \wedge a^{\prime \prime}, a^{\prime} \vee a^{\prime \prime}=a, a^{\prime} \vee n_{1}<1$ and $a^{\prime \prime} \vee n_{1}<1$. Since $a^{\prime}<\left.\right|_{\left(m_{1}\right)} b_{i}$ and $a^{\prime \prime}<\left.\right|_{\left(m_{1}\right)} b_{i}(i=1,2)$, by Lemma 2.5 (ii) there exist $b_{i}^{\prime}, b_{\imath}^{\prime \prime} \leqq b_{i}$ such that $a^{\prime} \|_{\left(m_{1}, n_{i}\right)} b_{i}^{\prime}$ and $a^{\prime \prime} \|_{\left(m_{1}, n_{i}\right)} b_{i}^{\prime \prime}$. Since $a \wedge\left(b_{i}^{\prime} \vee b_{i}^{\prime \prime}\right) \leqq$ $a \wedge b_{i}=0$ and

$$
a \vee n_{i}=a^{\prime} \vee a^{\prime \prime} \vee n_{i}=m_{1} \vee b_{i}^{\prime} \vee b_{i}^{\prime \prime},
$$

we have $a \|_{\left(m_{1}, n_{i}\right)} b_{i}^{\prime} \vee b_{i}^{\prime \prime}$. Hence $b_{i}=b_{i}^{\prime} \vee b_{i}^{\prime \prime}$ by (P 1). On the other hand, since $a^{\prime} \vee b_{1}^{\prime}=a^{\prime} \vee n_{1}<1$, it follows from (iii) that $b_{1}^{\prime} \|_{\left(n_{1}, n_{2}\right)} b_{2}^{\prime}$. Similarly, $b_{1}^{\prime \prime} \|_{\left(n_{1}, n_{2}\right)} b_{2}^{\prime \prime}$. Hence $b_{1} \|_{\left(n_{1}, n_{2}\right)} b_{2}$ by (P 2).

CoRollary. If $a\left\|_{(m, n)} b, b\right\|_{(n, r)} c$ and $c \|_{(r, m)} d$ where $m, n, r \in \mathscr{C}$ then $a=d$.

Proof. Since $b\left\|_{(n, r)} c, b\right\|_{(n, m)} a$ and $c \wedge m=0$, it follows from Theorem 4.2 that $c \|_{(r, m)} a$. Hence $a=d$ by (P 1$)$.

LEMMA 4.5. If $a\left\|_{\left(m, n_{1}\right)} b_{1}, b_{1}\right\|_{\left(n_{1}, r\right)} c_{1}, a \|_{\left(m, n_{2}\right)} b_{2}$ and $b_{2} \|_{\left(n_{2}, r\right)} c_{2}$ where $m, n_{1}, n_{2}, r \in \mathscr{C}$ then $c_{1}=c_{2}$.

Proof. (i) When $r \wedge a=0$, it follows from Theorem 4.2 that $a \|_{(m, r)} c_{1}$ and $a \|_{(m, r)} c_{2}$. Hence $c_{1}=c_{2}$ by (P 1).

(ii) When $n_{1} \leqq n_{2}$ and $r \leqq a$, then since $b_{1} \|_{\left(n_{1}, r\right)} b, b_{1}<\left.\right|_{\left(n_{1}\right)} a$ and $r \leqq a$, we have $c_{1} \leqq a$ by Lemma 4.1. Moreover we have $b_{1} \leqq b_{2}$, since $a \|_{\left(m, n_{1}\right)} b_{1}, a<\left.\right|_{(m)} b_{2}$ and $n_{1} \leqq n_{2} \leqq b_{2}$. Since $c_{1} \|_{\left(r, n_{1}\right)} b_{1}$ and $c_{1} \wedge b_{2} \leqq$ $a \wedge b_{2}=0$, we have $c_{1}<\left.\right|_{(r)} b_{2}$ by Lemma 2.2 (ii). Putting $b_{2}^{\prime}=b_{2} \wedge$ $\left(n_{2} \vee c_{1}\right)$, we have $c_{1} \|_{\left(r, n_{2}\right)} b_{2}^{\prime}$ by Lemma 2.5 (ii). Since $c_{1} \leqq a$ and $a \wedge b_{2}^{\prime} \leqq a \wedge b_{2}=0$, we have $b_{2}^{\prime}<\left.\right|_{\left(n_{2}\right)} a$ by Lemma 2.2 (ii). Moreover, since $b_{1} \leqq n_{1} \vee c_{1} \leqq n_{2} \vee c_{1}$, we have $b_{1} \leqq b_{2}^{\prime}$, and hence $a \leqq m \vee b_{1} \leqq$ $m \vee b_{2}^{\prime}$. Therefore $a \|_{\left(m, n_{2}\right)} b_{2}^{\prime}$. By (P 1), we have $b_{2}=b_{2}^{\prime}$, whence $b_{2} \|_{\left(n_{2}, r\right)} c_{1}$. By (P 1) again, we have $c_{1}=c_{2}$.

(iii) When $n_{1} \leqq n_{2}$, we put $r^{\prime}=r \wedge a$ and take $r^{\prime \prime}$ such that $r=r^{\prime} \vee r^{\prime \prime}$ and $r^{\prime} \wedge r^{\prime \prime}=0$. If $r^{\prime}=0$ or $r^{\prime \prime}=0$, then we have $c_{1}=c_{2}$ by (i) or (ii). Let $r^{\prime} \neq 0$ and $r^{\prime \prime} \neq 0$. Then $r^{\prime}, r^{\prime \prime} \in \mathscr{C}$. By Theorem 
4.1 there exist $c_{i}^{\prime}$ and $c_{i}^{\prime \prime}(i=1,2)$ such that $b_{i} \|_{\left(n_{i}, n^{\prime}\right)} c_{i}^{\prime}$ and $b_{i} \|_{\left(n_{i}, r^{\prime \prime}\right)} c_{i}^{\prime \prime}$. Then $b_{i} \|_{\left(n_{i}, r\right)} c_{i}^{\prime} \vee c_{i}^{\prime \prime}$ by Lemma 4.3. Hence $c_{i}=c_{i}^{\prime} \vee c_{i}^{\prime \prime}$ by (P 1). Since $r^{\prime} \leqq a$, it follows from (ii) that $c_{1}^{\prime}=c_{2}^{\prime}$, and since $r^{\prime \prime} \wedge a=0$, it follows from (i) that $c_{1}^{\prime \prime}=c_{2}^{\prime \prime}$. Hence $c_{1}=c_{2}$.

(iv) When $n_{1} \$ b_{2}$, then $n_{1} \wedge b_{2}<n_{1}$, and hence there exists $n_{1}^{\prime} \in \mathscr{C}$ such that $n_{1}^{\prime} \leqq n_{1}$ and $n_{1}^{\prime} \wedge b_{2}=0$. Putting $b_{1}^{\prime}=b_{1} \wedge\left(n_{1}^{\prime} \vee a\right)$, we have $a \|_{\left(m, n_{1}^{\prime}\right)} b_{1}^{\prime}$ by Lemma 2.5 (ii). Since $b_{1}^{\prime} \wedge r \leqq b_{1} \wedge r=0$, by Theorem 4.1 there exists $c_{1}^{\prime}$ such that $b_{1}^{\prime} \|_{\left(n_{1}^{\prime}, r\right)} c_{1}^{\prime}$. Since $n_{1}^{\prime} \leqq n_{1}$, we have $c_{1}^{\prime}=c_{1}$ by (iii). On the other hand, since $b_{2} \wedge n_{1}^{\prime}=0$, it follows from Theorem 4.2 that $b_{2} \|_{\left(n_{2}, n_{1}^{\prime}\right)} b_{1}^{\prime}$. Since $c_{2} \|_{\left(r, n_{2}\right)} b_{2}$ and $b_{1}^{\prime} \|_{\left(n_{1}^{\prime}, r\right)} c_{1}^{\prime}$, we have $c_{2}=c_{1}^{\prime}$ by Corollary of Theorem 4.2. Hence $c_{1}=c_{2}$. When $n_{2} \not \leq b_{1}$, then we have $c_{1}=c_{2}$ by the same way.

(v) When $n_{1} \leqq b_{2}$ and $n_{2} \leqq b_{1}$, we have $b_{1} \leqq b_{2}$ and $b_{2} \leqq b_{1}$ by Lemma 4.1. Hence $b_{1}=b_{2}$, which implies $c_{1}=c_{2}$ by (P 1$)$.

5. Parallel images of incomplete elements. Let $m \in \mathscr{C}$ and let $a$ be an incomplete element over $m$, that is, $a \in I_{m}$. For any $n \in \mathscr{C}$, we define the parallel image of $a$ at $n$, denoted by $\varphi_{n}(a)$, as follows:

(i) When $n \wedge a=0$, it follows from Theorem 4.1 that there exists a unique element $b$ such that $a \|_{(m, n)} b$. We define $\varphi_{n}(a)=b$.

(ii) When $n \leqq a$, there exists $n_{0} \in \mathscr{C}$ such that $n_{0} \wedge a=0$, since $a<1$. Then there exists $b$ such that $a \|_{\left(m, n_{0}\right)} b$, and since $b \wedge n \leqq$ $b \wedge a=0$, there exists $c$ such that $b \|_{\left(n_{0}, n\right)} c$. It follows from Lemma 4.5 that $c$ is independent of the choice of $n_{0}$. We define $\varphi_{n}(a)=c$. Note that we have $\varphi_{n}(a) \leqq a$ by Lemma 4.1 , since $b \|_{\left(n_{0}, n\right)} \varphi_{n}(a), b<\left.\right|_{\left(n_{0}\right)} a$ and $n \leqq a$.

(iii) When $n \wedge a \neq 0$ and $n \not a$, we have $n \wedge a \in \mathscr{C}$ and hence we get $\varphi_{n \wedge a}(a)$ by (ii). We define $\varphi_{n}(a)=\varphi_{n \wedge a}(a) \vee n$.

REMARK 5.1. Let $a \in I_{m}$. Evidently $\varphi_{m}(a)=a$, and $\varphi_{n}(a) \leqq a \vee n$ for every $n \in \mathscr{C}$. When $n \wedge a \neq 0$ and $n \leqq a$, putting $n_{1}=n \wedge a$, there exists $n_{2} \in \mathscr{C}$ such that $n=n_{1} \vee n_{2}$ and $n_{1} \wedge n_{2}=0$. Since $n_{2} \wedge a=0$, we have $\varphi_{n_{1}}(a) \|_{\left(n_{1}, n_{2}\right)} \varphi_{n_{2}}(a)$ by the definition of $\varphi_{n_{1}}(a)$. Hence $\varphi_{n_{1}}(a) \vee \varphi_{n_{2}}(a)=\varphi_{n_{1}}(a) \vee n_{2}=\varphi_{n_{1}}(a) \vee n=\varphi_{n}(a)$.

Lemma 5.1. Let $a \in I_{m}$ and $n_{1}, n_{2} \in \mathscr{C}$. If $\left(a \vee n_{1}\right) \wedge n_{2}=0$ then $\varphi_{n_{1}}(a) \|_{\left(n_{1}, n_{2}\right)} \varphi_{n_{2}}(a)$.

Proof. (i) When $n_{1} \wedge a=0$, we have $\left(a, n_{1}, n_{2}\right) \perp$. Since $a \|_{\left(m, n_{i}\right)} \varphi_{n_{i}}(a)(i=1,2)$, we have $\varphi_{n_{1}}(a) \|_{\left(n_{1}, n_{2}\right)} \varphi_{n_{2}}(a)$ by Lemma 4.2. When $n_{1} \leqq a$, then $\varphi_{n_{1}}(a) \|_{\left(n_{1}, n_{2}\right)} \varphi_{n_{2}}(a)$ by the definition of $\varphi_{n_{1}}(a)$.

(ii) When $n_{1} \wedge a \neq 0$ and $n_{1} \not a$, we put $n_{1}^{\prime}=n_{1} \wedge a$ and take $n_{1}^{\prime \prime}$ such that 


$$
n_{1}=n_{1}^{\prime} \vee n_{1}^{\prime \prime} \text { and } n_{1}^{\prime} \wedge n_{1}^{\prime \prime}=0 .
$$

By (i) we 'have $\varphi_{n_{1}^{\prime \prime}}(a) \|_{\left(n_{1}^{\prime \prime}, n_{2}\right)} \varphi_{n_{2}}(a)$ and $\varphi_{n_{1}^{\prime}}(a) \|_{\left(n_{1^{\prime}}, n_{2}\right)} \varphi_{n_{2}}(a)$. Now we shall show that $\varphi_{n_{2}}(a) \wedge n_{1}=0$. We have $n_{1}=n_{1}^{\prime} \vee n_{1}^{\prime \prime} \leqq a \vee n_{1}^{\prime \prime}$ and $\varphi_{n_{2}}(a) \leqq a \vee n_{2}$. Moreover $\left(a, n_{1}^{\prime \prime}, n_{2}\right) \perp$. Hence

$$
\varphi_{n_{2}}(a) \wedge n_{1} \leqq\left(a \vee n_{2}\right) \wedge\left(a \vee n_{1}^{\prime \prime}\right)=a \vee\left\{n_{2} \wedge\left(a \vee n_{1}^{\prime \prime}\right)\right\}=a .
$$

Therefore $\varphi_{n_{2}}(a) \wedge n_{1} \leqq \varphi_{n_{2}}(a) \wedge a=0$. By Remark 5.1, we have $\varphi_{n_{1}^{\prime}}(a) \vee \varphi_{n_{1}^{\prime}}(a)=\varphi_{n_{1}}(a)$. Hence we have $\varphi_{n_{1}}(a) \|_{\left(n_{1}, n_{2}\right)} \varphi_{n_{2}}(a)$ by Lemma 4.3.

Lemma 5.2. Let $a \in I_{m}(m \in \mathscr{C})$ and $n \in \mathscr{C}$. Then $n$ is a maximal element of $\mathscr{C}$ contained in $\varphi_{n}(a)$.

Proof. ( i ) When $n \wedge a=0$, we have $\Phi_{n}(a) \|_{(n, m)} a$. By Lemma 2.1, $n$ is maximal in the set $\left\{n^{\prime} \in \mathscr{C} ; n^{\prime} \leqq \varphi_{n}(a)\right\}$. Since $\mathscr{C} \subset \mathscr{L}, n$ is maximal in $\left\{n^{\prime} \in \mathscr{C} ; n^{\prime} \leqq \varphi_{n}(a)\right\}$.

(ii) When $n \leqq a$, taking $n_{0} \in \mathscr{C}$ with $a \wedge n_{0}=0$, we have $\varphi_{n}(a) \|_{\left(n: n_{0}\right)} \varphi_{n_{0}}(a)$. Hence $n$ is maximal as in (i).

(iii) When $n \wedge a \neq 0$ and $n \not a$, we put $n \wedge a=n_{1}$ and take $n_{2}$ such that $n=n_{1} \vee n_{2}$ and $n_{1} \wedge n_{2}=0$. Then $n_{1}, n_{2} \in \mathscr{C}$. Let $n^{\prime}$ be an element of $\mathscr{C}$ such that $n \leqq n^{\prime} \leqq \varphi_{n}(a)$. Since $\varphi_{n}(a)=\varphi_{n_{1}}(a) \vee n$ and $\varphi_{n_{1}}(a) \leqq a$, we have $n^{\prime} \wedge a \leqq\left(\varphi_{n_{1}}(a) \vee n\right) \wedge a=\varphi_{n_{1}}(a) \vee(n \wedge a)=$ $\varphi_{n_{1}}(a)$. Since $n_{1} \leqq n^{\prime} \wedge a \in \mathscr{C}$, we have $n^{\prime} \wedge a=n_{1}$ by (ii). If we had $n<n^{\prime}$, then there would exists $n_{0} \in \mathscr{C}$ such that $n_{0} \leqq n^{\prime}$ and $n_{0} \wedge n=0$. Since $\left(n_{1}, n_{2}, n_{0}\right) \perp$, we have

$$
a \wedge\left(n_{2} \vee n_{0}\right)=a \wedge n^{\prime} \wedge\left(n_{2} \vee n_{0}\right)=n_{1} \wedge\left(n_{2} \vee n_{0}\right)=0 .
$$

Hence $\left(a, n_{2}, n_{0}\right) \perp$. But $n_{0} \leqq n^{\prime} \leqq \Phi_{n}(a) \leqq a \vee n=a \vee n_{2}$, a contradiction. Therefore $n=n^{\prime}$, and hence $n$ is maximal.

Lemma 5.3. Let $a \in I_{m}(m \in \mathscr{C})$ and $n_{1} \leqq n$ where $n_{1}, n \in \mathscr{C}$. Then $\varphi_{n}(a)=\varphi_{n_{1}}(a) \vee n$.

Proof. We may assume $n_{1}<n$. Take $n_{2} \in \mathscr{C}$ such that $n=n_{1} \vee n_{2}$ and $n_{1} \wedge n_{2}=0$.

(i) When $n \wedge a=0$, we have $a \|_{(m, n)} \varphi_{n}(a)$ and $a \|_{\left(m, n_{i}\right)} \varphi_{n_{i}}(a)$ $(i=1,2)$. By Lemma 4.3 and (P 1) we have $\varphi_{n}(a)=\varphi_{n_{1}}(a) \vee \varphi_{n_{2}}(a)$. Since $n_{1} \leqq \varphi_{n_{1}}(a) \wedge n \leqq \varphi_{n_{1}}(a)$ and $\varphi_{n_{1}}(a) \wedge n \in \mathscr{C}$, we have $\varphi_{n_{1}}(a) \wedge n=$ $n_{1}$ by Lemma 5.2, and hence $\varphi_{n_{1}}(a) \wedge n_{2}=\varphi_{n_{1}}(a) \wedge n \wedge n_{2}=n_{1} \wedge n_{2}=0$. Hence $\varphi_{n_{1}}(\alpha) \|_{\left(n_{1}, n_{2}\right)} \varphi_{n_{2}}(\alpha)$ by Theorem 4.2. Therefore

$$
\varphi_{n}(a)=\varphi_{n_{1}}(a) \vee \varphi_{n_{2}}(a)=\varphi_{n_{1}}(a) \vee n_{2}=\varphi_{n_{1}}(a) \vee n \text {. }
$$

(ii) When $n \leqq a$, taking $n_{0} \in \mathscr{C}$ with $a \wedge n_{0}=0$, we have 
$\varphi_{n_{0}}(a) \|_{\left(n_{0}, n\right)} \varphi_{n}(a)$ and $\varphi_{n_{0}}(a) \|_{\left(n_{0}, n_{i}\right)} \varphi_{n_{i}}(a)(i=1,2)$. Hence by the same way as (i), we have $\varphi_{n}(a)=\varphi_{n_{1}}(a) \vee n$.

Hence

(iii) When $n \wedge a \neq 0$ and $n_{1} \wedge a=0$, we have $\varphi_{n \wedge a}(a) \|_{\left(n \wedge a, n_{1}\right)} \varphi_{n_{1}}(a)$.

$$
\begin{aligned}
\varphi_{n}(a) & =\varphi_{n \wedge a}(a) \vee n=\varphi_{n \wedge a}(a) \vee n_{1} \vee n=\varphi_{n_{1}}(a) \vee(n \vee a) \vee n \\
& =\varphi_{n_{1}}(a) \vee n .
\end{aligned}
$$

When $n_{1} \wedge a \neq 0$, it follows from (ii) that $\varphi_{n \wedge a}(a)=\varphi_{n_{1} \wedge a}(a) \vee(n \wedge a)$. Hence

$$
\rho_{n}(a)=\varphi_{n \wedge a}(a) \vee n=\varphi_{n_{1} \wedge a}(a) \vee n=\varphi_{n_{1} \wedge a}(a) \vee n_{1} \vee n=\varphi_{n_{1}}(a) \vee n .
$$

Theorem 5.1. Let $a \in I_{m}(m \in \mathscr{C})$ and $n \in \mathscr{C}$. The mapping $x \rightarrow$ $\varphi_{n}(x)$ is an isomorphism of the interval $L[m, a]$ onto $L\left[n, \varphi_{n}(a)\right]$.

Proof. (i) When $n \wedge a=0$, we have $a \|_{(m, n)} \varphi_{n}(a)$. For any $x \in L[m, a]$, we have $x \in I_{m}$ and $x \|_{(m, n)} \varphi_{n}(x)$. Hence $\varphi_{n}(x)=\varphi_{n}(a) \wedge$ $(n \vee x)$ by Lemma 2.5 (ii) and (P 1). It follows from Lemma 2.6 that $\varphi_{n}$ is an isomorphism of $L[m, a]$ onto $L\left[n, \varphi_{n}(a)\right]$.

(ii) When $n \leqq a$, taking $n_{0} \in \mathscr{C}$ with $n_{0} \wedge a=0$, we have $a \|_{\left(m, n_{0}\right)} \varphi_{n_{0}}(a)$ and $\varphi_{n_{0}}(a) \|_{\left(n_{0}, n\right)} \varphi_{n}(a)$. It follows from (i) and Lemma 2.6 that the composed mapping $x \rightarrow \varphi_{n_{0}}(x) \rightarrow \varphi_{n}(x) \wedge\left(n \vee \varphi_{n_{0}}(x)\right)$ is an isomorphism of $L[m, a]$ onto $L\left[n, \varphi_{n}(a)\right]$. On the other hand, since $(x \vee n) \wedge n_{0} \leqq a \wedge n_{0}=0$, we have $\varphi_{n}(x) \|_{\left(n, n_{0}\right)} \varphi_{n_{0}}(x)$ by Lemma 7.1. Hence $\varphi_{n}(x)=\varphi_{n}(a) \wedge\left(n \vee \varphi_{n_{0}}(x)\right)$ by $(\mathrm{P} 1)$.

(iii) When $n \wedge a \neq 0$ and $n \not a$, we put $n_{1}=n \wedge a$. Then we have $\varphi_{n}(a)=\varphi_{n_{1}}(a) \vee n$, and moreover $\varphi_{n_{1}}(a) \wedge n=n_{1}$ since $n_{1} \leqq$ $\varphi_{n_{1}}(a) \wedge n \leqq a \wedge n=n_{1}$. Since $L\left[n_{1}, 1\right]$ is modular, the mapping $y \rightarrow$ $y \vee n$ is an isomorphism of $L\left[n_{1}, \varphi_{n_{1}}(a)\right]$ onto $L\left[n, \varphi_{n}(a)\right]$. By (ii), the composed mapping $x \rightarrow \varphi_{n_{1}}(x) \rightarrow \varphi_{n_{1}}(x) \vee n$ is an isomorphism of $L[m, a]$ onto $L\left[n, \varphi_{n}(a)\right]$. Moreover $\varphi_{n_{1}}(x) \vee n=\varphi_{n}(x)$ by Lemma 5.3.

Lemma 5.4. Let $a \in I_{m}(m \in \mathscr{C})$ and $n=n_{1} \vee n_{2}$ where $n, n_{1}, n_{2} \in \mathscr{C}$. Then $\varphi_{n}(a)=\varphi_{n_{1}}(a) \vee \varphi_{n_{2}}(a)$.

Proof. Since $n=n_{1} \vee n_{2} \leqq \varphi_{n_{1}}(a) \vee \varphi_{n_{2}}(a)$, it follows from Lemma 5.3 that $\varphi_{n}(a)=\varphi_{n_{1}}(a) \vee n \vee \varphi_{n_{2}}(a) \vee n=\varphi_{n_{1}}(a) \vee \varphi_{n_{2}}(a)$.

THEOREM 5.2. If $a \|_{(m, n)} b$ where $m, n \in \mathscr{C}$ then $\varphi_{r}(a)=\varphi_{r}(b)$ for every $r \in \mathscr{C}$.

Proof. (i) When $r \leqq a$, we have $b \|_{(n, r)} \varphi_{r}(b)$ since $r \wedge b \leqq a \wedge$ $b=0$. On the other hand, since $n \wedge a=0$, we have $\varphi_{r}(a) \|_{(r, n)} b$. Hence $\varphi_{r}(a)=\varphi_{r}(b)$ by $(\mathrm{P} 1)$. When $r \leqq b$, similarly we have $\varphi_{r}(a)=\varphi_{r}(b)$. 
(ii) When $r \wedge a=r \wedge b=0$, we have $\varphi_{r}(a)\left\|_{(r, m)} a, a\right\|_{(m, n)} b$ and $B \|_{(n, r)} \varphi_{r}(b)$. Hence $\varphi_{r}(a)=\varphi_{r}(b)$ by Corollary Theorem 4.2.

(iii) When $r \wedge a=0$, we put $r_{1}=r \wedge b$ and take $r_{2}$ such that $r=r_{1} \vee r_{2}$ and $r_{1} \wedge r_{2}=0$. If $r_{1}=0$ or $r_{2}=0$ then $\varphi_{r}(a)=\varphi_{r}(b)$ holds by (ii) or (i). Hence we may assume $r_{1}, r_{2} \in \mathscr{C}$. Then $\varphi_{r}(a)=$ $\varphi_{r_{1}}(a) \vee \varphi_{r_{2}}(a)$ and $\varphi_{r}(b)=\varphi_{r_{1}}(b) \vee \varphi_{r_{2}}(b)$ by Lemma 5.4. We have $\varphi_{r_{i}}(a)=\varphi_{r_{i}}(b)(i=1,2)$ by (i) and (ii). Hence $\varphi_{r}(a)=\varphi_{r}(b)$.

(iv) When $r \wedge a \neq 0$ and $r \$ a$, we put $r_{1}=r \wedge a$ and take $r_{2}$ such that $r=r_{1} \vee r_{2}$ and $r_{1} \wedge r_{2}=0$. Then $r_{1}, r_{2} \in \mathscr{C}$, and then $\varphi_{r}(a)=\varphi_{r_{1}}(a) \vee \varphi_{r_{2}}(a)$ and $\varphi_{r}(b)=\varphi_{r_{1}}(b) \vee \varphi_{r_{2}}(b)$ by Lemma 5.4. We have $\varphi_{r_{i}}(a)=\varphi_{r_{i}}(b)$ by (i) and (iii). Hence $\varphi_{r}(a)=\varphi_{r}(b)$.

LEMMA 5.5. Let $a \in I_{m}(m \in \mathscr{C})$ and $n \in \mathscr{C}$. If $\varphi_{n}(a)<1$, then $\varphi_{n}(a) \in I_{n}$ and $\varphi_{r}\left(\varphi_{n}(\alpha)\right)=\varphi_{r}(\alpha)$ for every $r \in \mathscr{C}$.

Proof. ( i ) When $a \wedge n=0$, we have $a \|_{(m, n)} \varphi_{n}(a)$. Hence $\varphi_{n}(a) \in I_{n}$, and $\varphi_{r}(a)=\varphi_{r}\left(\varphi_{n}(a)\right)$ by Theorem 5.2. When $a \vee n<1$, we take $n_{0} \in \mathscr{C}$ such that $(a \vee n) \wedge n_{0}=0$. Then $\varphi_{n}(a) \|_{\left(n, n_{0}\right)} \varphi_{n_{0}}(a)$ by Lemma 5.1. Hence $\varphi_{n}(a) \in I_{n}$. Moreover, since $a \|_{\left(m, n_{0}\right)} \varphi_{n_{0}}(a)$, we have $\varphi_{r}(a)=\varphi_{r}\left(\varphi_{n_{0}}(a)\right)=\varphi_{r}\left(\varphi_{n}(a)\right)$ by Theorem 5.2.

(ii) When $a \wedge n \neq 0$ and $a \vee n=1$, we put $n_{1}=a \wedge n$ and take $n_{2}$ such that $n=n_{1} \vee n_{2}$ and $n_{1} \wedge n_{2}=0$. Since $a<1$, we have $n \geqq a$, and hence $n_{1}, n_{2} \in \mathscr{C}$. We have $\varphi_{n_{1}}(a)<a$, since otherwise $\varphi_{n_{1}}(a)=a$ and then $\varphi_{n}(a)=\varphi_{n_{1}}(a) \vee n=a \vee n=1$, a contradiction. Hence there exists $n_{0} \in \mathscr{C}$ such that $n_{0} \leqq a$ and $\varphi_{n_{1}}(a) \wedge n_{0}=0$. Since $n_{1}, n_{0} \leqq a$ and $a \wedge n_{2}=0$, we have $\varphi_{n_{1}}(\alpha) \|_{\left(n_{1}, n_{2}\right)} \varphi_{n_{2}}(\alpha)$ and $\varphi_{n_{0}}(\alpha) \|_{\left(n_{0}, n_{2}\right)} \varphi_{n_{2}}(\alpha)$. Moreover $\varphi_{n_{1}}(a) \|_{\left(n_{1}, n_{0}\right)} \varphi_{n_{0}}(a)$ by Theorem 4.2. Since $\left(\varphi_{n_{0}}(a) \vee n_{1}\right) \wedge n_{2} \leqq$ $a \wedge n_{2}=0$, we have $\left(\varphi_{n_{0}}(\alpha), n_{1}, n_{2}\right) \perp$, and hence $\varphi_{n_{0}}(a) \wedge\left(n_{1} \vee n_{\vartheta}\right)=0$. Hence, by Lemma 4.3 , we have $\varphi_{n_{0}}(a) \|_{\left(n_{0}, n\right)} \varphi_{n_{1}}(a) \vee \varphi_{n_{2}}(a)=\varphi_{n}(a)$. Therefore $\varphi_{n}(a) \in I_{n}$. Since $a \|_{\left(m_{1}, n_{2}\right)} \varphi_{n_{2}}(a)$, we have $\varphi_{r}(a)=\varphi_{r}\left(\mathscr{P}_{n_{2}}(a)\right)=$ $\varphi_{r}\left(\varphi_{n_{0}}(a)\right)=\varphi_{r}\left(\varphi_{n}(a)\right)$ by Theorem 5.2.

LEMMA 5.6. Let $a \in I_{m}(m \in \mathscr{C})$ and $n_{1}, n_{2} \in \mathscr{C}$. If $\varphi_{n_{1}}(a) \wedge n_{2}=0$ then $\varphi_{n_{1}}(\alpha) \|_{\left(n_{1}, n_{2}\right)} \varphi_{n_{2}}(\alpha)$.

Proof. Since $\varphi_{n_{1}}(a)<1$, we have $\varphi_{n_{1}}(a) \in I_{n_{1}}$ by Lemma 5.5. Hence $\mathcal{P}_{n_{2}}\left(\varphi_{n_{1}}(a)\right) \|_{\left(n_{2}, n_{1}\right)} \varphi_{n_{1}}(a)$. By Lemma 5.5, we have $P_{n_{2}}\left(\varphi_{n_{1}}(a)\right)=\varphi_{n_{2}}(a)$.

DEFINITION. Let $\mathscr{C}_{0}$ be the subset of $\mathscr{C}$ deleting maximal elements in $\mathscr{C}$ which are not atoms, that is, $\mathscr{C}_{0}=(\mathscr{C}-\{$ maximal elements $\}) \smile \Omega$. Then, it is evident that for any $m \in \mathscr{C}$ there exists $m_{1} \in \mathscr{C}_{0}$ such that $m_{1} \leqq m$. Hence $L$ is $\mathscr{C}_{0}-S S C$.

Lemma 5.7. If $a \in I_{m}(m \in \mathscr{C})$ and $n \in \mathscr{C}_{0}$, then $\varphi_{n}(a) \in I_{n}$. 
Proof. If we had $\varphi_{n}(a)=1$, then $n$ would be maximal in $\mathscr{C}$ by Lemma 5.2. Hence $n$ would be an atom by the definition of $\mathscr{C}_{0}$. If $a \wedge n=0$, then $a \|_{(m, n)} \varphi_{n}(a)=1$, a contradiction. If $a \wedge n \neq 0$, then $n \leqq a$, and then $1=\varphi_{n}(a) \leqq a \in I_{m}$, a contradiction. Therefore, we have $\varphi_{n}(a)<1$, and hence $\varphi_{n}(a) \in I_{n}$ by Lemma 5.5 .

LEMmA 5.8. If $m, n \in \mathscr{C}_{0}$, then $I_{m}$ and $I_{n}$ are isomorphic by mutually inverse mappings $\varphi_{n}$ and $\varphi_{m}$.

Proof. We have $\varphi_{n}\left(I_{m}\right) \subset I_{n}$ and $\varphi_{m}\left(I_{n}\right) \subset I_{m}$ by Lemma 5.7. It follows from Lemma 5.5 that $\varphi_{m}\left(\varphi_{n}(a)\right)=\varphi_{m}(a)=a$ for $a \in I_{m}$ and $\varphi_{n}\left(\varphi_{m}(b)\right)=\varphi_{n}(b)=b$ for $b \in I_{n}$. Moreover $\varphi_{m}$ and $\varphi_{n}$ are order-preserving by Theorem 5.1. Hence $I_{m}$ and $I_{n}$ are isomorphic.

Lemma 5.9. Let $m \in \mathscr{C}$. If $m$ is not an atom and if there exist $a_{1}, a_{2} \in I_{m}$ such that $a_{1} \vee a_{2}=1$, then $m \in \mathscr{C}_{0}$.

Proof. Since $L$ is left complemented, there exists $b \leqq a_{2}$ such that $a_{1} \vee b=a_{1} \vee a_{2}$ and $a_{1} \perp b$. Putting $a_{2}^{\prime}=m \vee b$, we have

$$
a_{2}^{\prime} \in I_{m}, a_{1} \vee a_{2}^{\prime}=a_{1} \vee b=a_{1} \vee a_{2}=1
$$

and

$$
a_{1} \wedge a_{2}^{\prime}=(m \vee b) \wedge a_{1}=m \vee\left(b \wedge a_{1}\right)=m .
$$

Hence we may assume that $a_{1} \wedge a_{2}=m$. Since $m$ is not an atom, there exists $m_{1} \in \mathscr{C}$ such that $m_{1} \leqq m$. Since $\varphi_{m_{1}}\left(a_{1}\right) \leqq a_{1}$, we have

$$
\varphi_{m_{1}}\left(a_{1}\right) \wedge a_{2}=\varphi_{m_{1}}\left(a_{1}\right) \wedge a_{1} \wedge a_{2}=\varphi_{m_{1}}\left(a_{1}\right) \wedge m=m_{1}
$$

by Lemma 5.2. Since $L$ is weakly modular and $\varphi_{m_{1}}\left(a_{1}\right) \wedge a_{2} \neq 0$, we have $\left(\varphi_{m_{1}}\left(a_{2}\right) \vee \varphi_{m_{1}}\left(a_{1}\right)\right) \wedge a_{2}=\varphi_{m_{1}}\left(a_{2}\right) \vee\left(\varphi_{m_{1}}\left(a_{1}\right) \wedge a_{2}\right)=\varphi_{m_{1}}\left(a_{2}\right) \wedge m_{1}=$ $\varphi_{m_{1}}\left(a_{2}\right)$. Since $\varphi_{m_{1}}\left(a_{2}\right) \wedge m=m_{1}$, we have $\varphi_{m_{1}}\left(a_{2}\right)<a_{2}$, and hence the above equation implies that $\varphi_{m_{1}}\left(a_{1}\right) \vee \varphi_{m_{1}}\left(a_{2}\right)<1$. Putting

$$
a_{0}=\varphi_{m_{1}}\left(a_{1}\right) \vee \varphi_{m_{1}}\left(a_{2}\right) \text {, }
$$

we have $a_{0} \in I_{m_{1}}$ by Corollary of Theorem 4.1, and by Theorem 5.1 we have

$$
\begin{aligned}
\varphi_{m}\left(a_{0}\right) & =\varphi_{m} \varphi_{m_{1}}\left(a_{1}\right) \vee \varphi_{m} \varphi_{m_{1}}\left(a_{2}\right)=\varphi_{m}\left(a_{1}\right) \vee \varphi_{m}\left(a_{2}\right)=a_{1} \vee a_{2} \\
& =1 \in I_{m} .
\end{aligned}
$$

By Lemma 5.7, we have $m \in \mathscr{C}_{0}$.

LEMMA 5.10. If for some $m \in \mathscr{C}_{0}$ there exist $a_{1}, a_{2} \in I_{m}$ such that $a_{1} \vee a_{2}=1$, then every element $n$ of $\mathscr{C}_{0}$ is an atom (hence $L$ is ato-mistic) and $\varphi_{n}\left(a_{1}\right) \vee \varphi_{n}\left(a_{2}\right)=1$. 
Proof. It follows from Lemma 5.9 that $m$ is an atom. Let $n \in \mathscr{C}_{0}$. If we had $\varphi_{n}\left(a_{1}\right) \vee \varphi_{n}\left(a_{2}\right)<1$, then as in the proof of Lemma 5.9, we would have $m \notin \mathscr{C}_{0}$. Hence $\varphi_{n}\left(a_{1}\right) \vee \varphi_{n}\left(a_{2}\right)=1$, and then $n$ is an atom by Lemma 5.9 .

6. Construction of modular extensions. As in $\S \S 4$ and 5, let $L$ be a weakly modular, left complemented lattice, with 0 and 1 , of length $\geqq 4$, and assume that for fixed subset $\mathscr{C}$ of $\mathscr{C}$, satisfying (C 1), $L$ is $\mathscr{C}-S S C$ and $L$ satisfies the axioms (P 1) and (P 2) with respect to $\mathscr{C}$. (We may assume that $\mathscr{C}$ satisfies (C 2).)

Let $\mathscr{C}_{0}$ be the subset of $\mathscr{C}$ given in $\S 5$. We say that $L$ is of type $A$ when for some $m \in \mathscr{C}_{0}$ there exist $a_{1}, a_{2} \in I_{m}$ such that $a_{1} \vee$ $a_{2}=1$. It follows from Lemma 5.8, Corollary of Theorem 4.1 and Lemma 5.10 that we have the following results.

(1) For any $m, n \in \mathscr{C}_{0}, I_{m}$ and $I_{n}$ are isomorphic by the mappings $\varphi_{n}$ and $\varphi_{m}$.

(2) If $L$ is not of type $A$ then $I_{m}$ is an ideal of the lattice $L[m, 1]$ for every $m \in \mathscr{C}_{0}$.

(3) If $L$ is of type $A$ then $L$ is atomistic and $\mathscr{C}_{0}=\Omega$. If moreover $L$ satisfies the axiom (P 3) with respect to $\Omega$ then $I_{m}=L[m, 1]-1$ for every $m \in \mathscr{C}_{0}$.

Hereafter, whenever $L$ is of type $A$, we assume that $L$ satisfies (P 3) with respect to $\Omega$. In this case, it is convenient that we set $1 \in I_{m}$ and $\varphi_{m}(1)=1$ for every $m \in \mathscr{C}_{0}$. Then $I_{m}=L[m, 1]$ and moreover $I_{m}$ and $I_{n}$ are isomorphic by $\varphi_{n}$ and $\varphi_{m}$.

Definition. For an incomplete element $a$, we denote by $[a]$ the set of parallel images of $a$ at all elements of $\mathscr{C}_{0}$, that is, $[a]=\left\{\mathscr{\rho}_{m}(a)\right.$; $\left.m \in \mathscr{C}_{0}{ }_{0}\right\}$. We denote by $S$ the set of all $[a]$ deleting $[m]$. For $[a]$, $[b] \in S$, we define $[a] \leqq[b]$ by $\Phi_{m}(a) \leqq \varphi_{m}(b)$ for some $m \in \mathscr{C}_{0}$ (and hence for every $\left.m \in \mathscr{C}_{0}\right)$. Hence $S$ is isomorphic to $I_{m}-\{m\}$ for every $m \in \mathscr{C}_{0}$. If $L$ is of type $A$, then $S$ has the greatest element [1] and $S \cong L[m, 1]-\{m\}$.

LEMmA 6.1. In the set $A \equiv L \smile S$, we define a partial order by the following conventions:

(O 1) For $a, b \in L$, we have $a \leqq b$ in $A$ when $a \leqq b$ in $L$. For $[a],[b] \in S$, we have $[a] \leqq[b]$ in $A$ when $[a] \leqq[b]$ in $S$.

(O 2) For $[a] \in S$ and $b \in L$, we have $[a]<b$ when $\varphi_{m}(a) \leqq b$ for $m \in \mathscr{C}_{0}$ with $m \leqq b$. (Especially, [1] $<b$ only when $b=1$.)

(O 3) For $[a] \in S$ and $b \in L$, we have $b<[a]$ only when $b=0$.

Then $A$ is a lattice where the lattice operations $U$ and $\cap$ have the following properties:

(1) If $a, b \in L$ then $a \cup b=a \vee b$. 
(2) If $0 \neq a \in L$ and $[b] \in S$ then for $m \in \mathscr{C}_{0}$ with $m \leqq a$ we have $a \cup[b]=a \vee \varphi_{m}(b)$.

(3) If $[a],[b] \in S$ then for $m \in \mathscr{C}_{0}$ we have $[a] \cup[b]=\left[\varphi_{m}(a) \vee\right.$ $\left.\varphi_{m}(b)\right]$.

(4) If $a, b \in L$ and $a \wedge b \neq 0$ then $a \cap b=a \wedge b$.

(5) If $a, b \in L$ and $a \wedge b=0$ and if $m, n \in \mathscr{C}_{0}$ such that $m \leqq a$ and $n \leqq b$ then $a \cap b=[a \wedge(m \vee b)]=[b \wedge(n \vee a)]$.

(6) If $a \in L,[b] \in S$ and if $m \in \mathscr{C}_{0}$ such that $m \leqq a$ then $a \cap[b]=$ $\left[a \wedge \varphi_{m}(b)\right]$.

(7) If $[a],[b] \in S$ and $m \in \mathscr{C}_{0}$ then $[a] \cap[b]=\left[\varphi_{m}(a) \wedge \varphi_{m}(b)\right]$. Note that in (5), (6) and (7) we set $0=[\mathrm{m}]$ for convenience. (If $L$ is of type $A$ then especially we have $a \cup[1]=1,[a] \cup[1]=[1], a \cap[1]=$ $[a]$ and $[a] \cap[1]=[a]$.)

Proof. (1) Let $a, b \in L$. To prove (1), we may assume $a \neq 0$ and $b \neq 0$. By $(03)$, any upper bound of $\{a, b\}$ in $\Lambda$ belongs to $L$. Hence $a \cup b$ exists and (1) holds.

(2) We have $[b]<\varphi_{m}(b)$ by $(02)$. Hence $a \vee \varphi_{m}(b)$ is an upper bound of $\{a,[b]\}$ in $\Lambda$. If $c$ is an upper bound of $\{a,[b]\}$ then $c \in L$ by (O 3). Since $m \leqq a \leqq c$ and $[b]<c$, we have $\varphi_{m}(b) \leqq c$. Thus $a \cup[b]$ exists and (2) holds.

(3) This follows from (O 1) evidently.

(4) Take $m \in \mathscr{C}_{0}$ such that $m \leqq a \wedge b$. If $c \in L$ is a lower bound of $\{a, b\}$ in $A$ then $c \leqq a \wedge b$ by $(01)$. If $[d] \in S$ is a lower bound of $\{a, b\}$ in $\Lambda$, then $\varphi_{m}(d) \leqq a \wedge b$ and hence $[d] \leqq a \wedge b$. Therefore $a \cap b$ exists and (4) holds.

(5) We have $a \wedge(m \vee b) \|_{\langle m, n)} b \wedge(n \vee a)$ by Lemma 2.5 (i). Hence, $[a \wedge(m \vee b)]=[b \wedge(n \vee a)]$ is a lower bound of $\{a, b\}$. Since $a \wedge b=0$, any lower bound is either an element of $S$ or 0 , and hence it has the form $[c]$ where $c \geqq m$. Since $[c] \leqq a, b$, we have $\varphi_{m}(c) \leqq a$ and $\varphi_{n}(c) \leqq b$. Since $\varphi_{m}(c) \wedge n \leqq a \wedge b=0$, we have $\varphi_{m}(c) \|_{(m, n)} \varphi_{n}(c)$ by Lemma 5.6. Hence $\varphi_{m}(c) \leqq a \wedge\left(m \vee \varphi_{n}(c)\right) \leqq a \wedge(m \vee b)$. Therefore $[c] \leqq[a \wedge(m \vee b)]$. Thus $a \cap b$ exists and (5) holds.

(6) It is evident that $\left[a \wedge \varphi_{m}(b)\right]$ is a lower bound of $\{a,[b]\}$. Any lower bound of $\{a,[b]\}$ has the form $[c]$ where $c \geqq m$. We have $[c] \leqq\left[a \wedge \varphi_{m}(b)\right]$ since $\varphi_{m}(c) \leqq a, \varphi_{m}(b)$. Hence $a \cap[b]$ exists and (6) holds.

(7) For $[a],[b] \in S$, taking $m \in \mathscr{C}_{0},\left[\varphi_{m}(a) \wedge \varphi_{m}(b)\right]$ is a lower bound of $\{[a],[b]\}$. For any lower bound $[c]$ of $\{[a],[b]\}$, we have $[c] \leqq\left[\varphi_{m}(a) \wedge \varphi_{m}(b)\right]$, since $\varphi_{m}(c) \leqq \varphi_{m}(a), \varphi_{m}(b)$. Hence $[a] \cap[b]$ exists and (7) holds.

LEMMA 6.2. The lattice 1 constructed in Lemma 6.1 is complemented. 
Proof. (i ) Let $0 \neq a \in L$. Since $L$ is left complemented, there exists $b \in L$ such that $a \vee b=1$ and $a \perp b$. We have $a \cup b=a \vee b=1$ by Lemma 6.1 (1). We take $m \in \mathscr{C}_{0}$ with $m \leqq a$. Since $(b, a) M$, we have $a \wedge(m \vee b)=(m \vee b) \wedge a=m \vee(b \wedge a)=m$. Hence, by Lemma $6.1(5), a \cap b=[a \wedge(m \vee b)]=[m]=0$. Therefore, $b$ is a complement of $a$ in $A$.

(ii) Let $[a] \in S$. We take $m \in \mathscr{C}_{0}$ with $m \leqq a$. Since $L$ is left complemented, it is relatively complemented. Hence there exists $b \in L$ such that $a \vee b=1$ and $a \wedge b=m$. By Lemma 6.1 (2), we have $[a] \cup b=\varphi_{m}(a) \vee b=a \vee b=1$. By Lemma 6.1 (6), we have $[a] \cap b=$ $\left[\varphi_{m}(a) \wedge b\right]=[a \wedge b]=[m]=0$. Hence $b$ is a complement of $[a]$ in $A$. (Especially, if $L$ is of type $A$, then $m$ is a complement of [1].)

Lemma 6.3. The lattice 1 constructed in Lemma 6.1 is modular.

Proof. (i) Let $a, b \in L$ and we shall show $(a, b) M$ in $\Lambda$. When $a \wedge b \neq 0$, we have $a \cap b=a \wedge b$ by Lemma 6.1 (4), and hence the interval $\Lambda[a \cap b, 1]$ of $A$ coinsides with $L[a \wedge b, 1]$ which is modular since $L$ is weakly modular. Hence $(a, b) M$ in $\Lambda[a \cap b, 1]$ and then $(a, b) M$ in $\Lambda$. When $a \wedge b=0$, we may assume $a \neq 0$ and $b \neq 0$. Let $\lambda$ be an element of $\Lambda$ with $0<\lambda<b$. If $\lambda \in L$, then we put $\lambda=b_{1}$ and take $n \in \mathscr{C}_{0}$ with $n \leqq b_{1}$. Since $(n \vee a) \wedge b \geqq n>0$ and since $L$ is weakly modular, we have $(n \vee a, b) M$ in $L$. Hence

$$
\begin{aligned}
\left(b_{1} \cup a\right) \cap b & =\left(b_{1} \vee a\right) \cap b=\left(b_{1} \vee a\right) \wedge b=\left(b_{1} \vee n \vee a\right) \wedge b \\
& =b_{1} \vee\{(n \vee a) \wedge b\} .
\end{aligned}
$$

On the other hand, by Lemma 6.1 (5) and (2), we have

$$
b_{1} \cup(a \cap b)=b_{1} \cup[b \wedge(n \vee a)]=b_{1} \vee\{b \wedge(n \vee a)\}
$$

Hence $\left(b_{1} \cup a\right) \cap b=b_{1} \cup(a \cap b)$.

If $\lambda \in S$, then we put $\lambda=[c]$ and take $m, n \in \mathscr{C}_{0}$ with $m \leqq a$ and $n \leqq b$. We shall prove $\left(\varphi_{m}(c) \vee a\right) \wedge b=0$. Since $[c]<b$, we have $\varphi_{n}(c) \leqq b$, whence $m \wedge \varphi_{n}(c) \leqq a \wedge b=0$. Hence $\varphi_{m}(c) \|_{(m, n)} \varphi_{n}(c)$ by Lemma 5.6, while $a \wedge(m \vee b) \|_{(m, n)} b \wedge(n \vee a)$ by Lemma 2.5 (i). Since $\left(\varphi_{n}(c) \vee\{b \wedge(n \vee a)\}\right) \wedge m \leqq b \wedge a=0$, by (P 2) we have

$$
\varphi_{m}(c) \vee\{a \wedge(m \vee b)\} \|_{(m, n)} \varphi_{n}(c) \vee\{b \wedge(n \vee a)\} .
$$

Now we have $(a, m \vee b) M$ since $L$ is weakly modular. Since $\varphi_{m}(c) \leqq$ $m \vee \varphi_{n}(c) \leqq m \vee b$, we have

$$
\varphi_{m}(c) \vee\{a \wedge(m \vee b)\}=\left(\varphi_{m}(c) \vee a\right) \wedge(m \vee b)
$$

Moreover, since $(n \vee a, b) M$, we have 


$$
\varphi_{n}(c) \vee\{b \wedge(n \vee a)\}=\left(\varphi_{n}(c) \vee n \vee a\right) \wedge b=\left(\varphi_{n}(c) \vee a\right) \wedge b .
$$

Hence, by $\left(^{*}\right)$, we have $0=\left(\varphi_{m}(c) \vee a\right) \wedge(m \vee b) \wedge\left(\varphi_{n}(c) \vee a\right) \wedge b=$ $\left(\varphi_{m}(c) \vee a\right) \wedge\left(\varphi_{n}(c) \vee a\right) \wedge b$. Since

$$
\varphi_{m}(c) \vee a \leqq m \vee \varphi_{n}(c) \vee a=\varphi_{n}(c) \vee a,
$$

we get $\left(\varphi_{m}(c) \vee a\right) \wedge b=0$. By Lemma 6.1 (2) and (5), we have

$$
\begin{aligned}
([c] \cup a) \cap b & =\left(\varphi_{m}(c) \vee a\right) \cap b=\left[\left(\varphi_{m}(c) \vee a\right) \wedge(m \vee b)\right] \\
& =\left[\varphi_{m}(c) \vee\{a \wedge(m \vee b)\}\right] .
\end{aligned}
$$

On the other hand, by Lemma 6.1 (5) and (3),

$$
[c] \cup(a \cap b)=[c] \cup[a \wedge(m \vee b)]=\left[\varphi_{m}(c) \vee\{a \wedge(m \vee b)\}\right] .
$$

Hence $([c] \cup a) \cap b=[c] \cup(a \cap b)$. Therefore, $(\lambda \cup a) \cap b=\lambda \cup(a \cap b)$ for any $\lambda \in A$ with $\lambda \leqq b$.

(ii) Let $[a] \in S$ and $b \in L$, and we shall show $([a], b) M$. We may assume $b \neq 0$. Let $\lambda \in \Lambda$ with $0<\lambda<b$. If $\lambda=b_{1} \in L$, then we take $n \in \mathscr{C}_{0}$ with $n \leqq b_{1}$. Since $\varphi_{n}(a) \wedge b \neq 0$, we have

$$
\left(b_{1} \cup[a]\right) \cap b=\left(b_{1} \vee \varphi_{n}(a)\right) \cap b=\left(b_{1} \vee \varphi_{n}(a)\right) \wedge b=b_{1} \vee\left(\varphi_{n}(a) \wedge b\right) \text {, }
$$

while by Lemma 6.1 (6) we have

$$
b_{1} \cup([a] \cap b)=b_{1} \cup\left[\varphi_{n}(a) \wedge b\right]=b_{1} \vee\left(\varphi_{n}(a) \wedge b\right) .
$$

If $\lambda=[c] \in S$, then we take $n \in \mathscr{C}_{0}$ with $n \leqq b$. Since $\varphi_{n}(c) \leqq b$, we have

$$
\begin{aligned}
([c] \cup[a]) \cap b & =\left[\varphi_{n}(c) \vee \varphi_{n}(a)\right] \cap b=\left[\left(\varphi_{n}(c) \vee \varphi_{n}(a)\right) \wedge b\right] \\
& =\left[\varphi_{n}(c) \vee\left(\varphi_{n}(a) \wedge b\right)\right],
\end{aligned}
$$

while

$$
[c] \cup([a] \cap b)=[c] \cup\left[\varphi_{n}(a) \wedge b\right]=\left[\varphi_{n}(c) \vee\left(\varphi_{n}(a) \wedge b\right)\right] .
$$

Hence $(\lambda \cup[a]) \cap b=\lambda \cup([a] \cap b)$ for any $\lambda \in \Lambda$ with $\lambda \leqq b$.

(iii) Let $a \in L$ and $[b] \in S$, and we shall show $(a,[b]) M$. We may assume $a \neq 0$. Let $\lambda \in \Lambda$ with $0<\lambda<[b]$. Then, since $\lambda \in S$, we put $\lambda=[c]$. We take $m \in \mathscr{C}_{0}$ with $m \leqq a$. Since $\varphi_{m}(c) \leqq \varphi_{m}(b)$, we have

$$
\begin{aligned}
([c] \cup a) \cap[b] & =\left(\varphi_{m}(c) \vee a\right) \cap[b]=\left[\left(\varphi_{m}(c) \vee a\right) \wedge \varphi_{m}(b)\right] \\
& =\left[\varphi_{m}(c) \vee\left(a \wedge \varphi_{m}(b)\right)\right],
\end{aligned}
$$

while

$$
[c] \cup(a \cap[b])=[c] \cup\left[a \wedge \varphi_{m}(b)\right]=\left[\varphi_{m}(c) \vee\left(a \wedge \varphi_{m}(b)\right)\right] .
$$

(iv) Let $[a],[b] \in S$ and let $[c]<[b]$. We take $m \in \mathscr{C}_{0}$ with $m \leqq a$. Since $\varphi_{m}(c) \leqq \varphi_{m}(b)$, we have 


$$
\begin{aligned}
([c] \cup[a]) \cap[b] & =\left[\varphi_{m}(c) \vee a\right] \cap[b]=\left[\left(\varphi_{m}(c) \vee a\right) \wedge \varphi_{m}(b)\right] \\
& =\left[\varphi_{m}(c) \vee\left(a \wedge \varphi_{m}(b)\right)\right],
\end{aligned}
$$

while

$$
[c] \cup([a] \cap[b])=[c] \cup\left[a \wedge \varphi_{m}(b)\right]=\left[\varphi_{m}(c) \vee\left(a \wedge \varphi_{m}(b)\right)\right] .
$$

Hence $([a],[b]) M$.

REMARK 6.1. We shall show that if an $\mathscr{C}$-SSC lattice, with 0 and 1 , has a finite connected chain from 0 to 1 , then it is atomistic. It suffices to prove that any nonzero element a includes an atom, since the lattice is SSC. Let $0=a_{0} \prec a_{1} \prec \cdots \prec a_{n}=1$ and let $r(<n)$ be greatest such that $a \geqq a_{r}$. Since $a \wedge a_{r}<a$, there exists $m \in \mathscr{C l}$ such that $m \leqq a\left(\leqq a_{r+1}\right)$ and $m \wedge a_{r}=0$. If $0<x \leqq m$, then $a_{r}<a_{r} \vee x \leqq$ $a_{r+1}$, whence $a_{r} \vee x=a_{r+1}$. Hence

$$
m=m \wedge a_{r+1}=\left(x \vee a_{r}\right) \wedge m=x \vee\left(a_{r} \wedge m\right)=x .
$$

Therefore $m$ is an atom included in $a$.

THEOREM 6.1. (Non-atomistic case) Let $L$ be an $\mathscr{C l}_{s}$-SSC lattice with 0 and 1 which is not atomistic. Then, $L$ is a Wilcox lattice if and only if $L$ is weakly modular, left complemented and satisfies two parallel axioms (P 1) and (P 2) with respect to $\mathscr{L l}_{s}$.

Proof. If $L$ is a Wilcox lattice, then evidently $L$ is weakly modular and it is left complemented by Lemma 3.2. Moreover $L$ satisfies the parallel axioms by Lemma 3.7. We shall prove the converse statement. We remark that $L$ is of infinite length by Remark 6.1 and that $L$ is not of type $A$. Putting $\mathscr{C}=\mathscr{M}_{s}, L$ satisfies all the conditions stated at the beginning of this section. Hence it follows from Lemmas 6.1, 6.2 and 6.3 that $L$ is a Wilcox lattice.

Theorem 6.2. (Atomistic case) Let $L$ be an atomistic lattice, with 0 and 1 , of length $\geqq 4$. Then, $L$ is a Wilcox lattice if and only if $L$ is weakly modular, left complemented and satisfies three parallel axioms (P 1), (P 2) and (P 3) with respect to $\Omega$.

Proof. As the proof of Theorem 6.1, the "only if" part follows from Lemmas 3.2 and 3.7 and the "if" part follows from Lemmas 6.1, 6.2 and 6.3 , by putting $\mathscr{C}=\Omega$.

REMARK 6.2. We remark that two axioms (P 2) and (P 3) can be replaced by the following one axiom (assuming that $L$ satisfies (P 1)): (P 4) If $a_{1}<\left.\right|_{(m)} b_{1}, a_{2}<\left.\right|_{(m)} b_{2}$ where $m \in \mathscr{C}$ and if $m<a \leqq a_{1} \vee a_{2}$ 
then for any $n \in \mathscr{C}$ with $a \wedge n=0$ there exists $b$ such that $a \|_{(m, n)} b$.

Proof. Since $L$ is $\mathscr{C}-S S C$, it is evident that (P 4) implies (P 3). We shall show that (P 4) implies (P 2). Let $a_{i} \|_{(m, n)} b_{i}(i=1,2)$ and $\left(a_{1} \vee a_{2}\right) \wedge n=0$. By (P 4) there exists $b$ such that $a_{1} \vee a_{2} \|_{(m, n)} b$. Then, $a_{i} \|_{(m, n)} b \wedge\left(n \vee a_{i}\right)$ by Lemma 2.5 (ii), whence $b_{i}=b \wedge\left(n \wedge a_{i}\right)$ by (P 1). Therefore, $b_{1} \vee b_{2} \leqq b$, and hence $\left(a_{1} \vee a_{2}\right) \wedge\left(b_{1} \vee b_{2}\right)=0$. On the other hand, we have $a_{1} \vee a_{2} \vee n=m \vee b_{1} \vee b_{2}$. Hence $a_{1} \vee$ $a_{2} \|_{(m, n)} b_{1} \vee b_{2}$. Conversely, we assume that (P 2) and (P 3) are satisfied. Then we may use the results in $\S 4$. Let $a_{i}<\left.\right|_{(m)} b_{i}(i=1,2), m<a \leqq$ $a_{1} \vee a_{2}$ and $a \wedge n=0$. If $a_{1} \vee a_{2}=1$ then $a \in I_{m}$ by (P 3). If $a_{1} \vee$ $a_{2}<1$ then $a_{1} \vee a_{2} \in I_{m}$ by Corollary of Theorem 4.1 and hence $a \in I_{m}$. Therefore it follows from Theorem 4.1 that there exists $b$ such that $a \|_{(m, n)} b$.

REMARK 6.3. We can show that an application of Theorem 6.2 to the upper continuous atomistic case implies the theorem on affine matroid lattices given in [2], p. 314. A matroid lattice is defined as an upper continuous atomistic lattice with the covering property ([4], Definition 1.8), which is left complemented and $M$-symmetric (see [8]). In an matroid lattice we write $a<\mid b$ when $a<\left.\right|_{(p)} b$ for some atom $p \leqq a$, and write $a|| b$ when $a<\mid b$ and $b<\mid a$. (In [2], p. 272, it is written $b \| a$ instead of $a<\mid b$.) A weakly modular matroid lattice $L$ of length $\geqq 4$ is called an affine matroid lattice ([4], Definition 3.3) when $L$ satisfies the following axiom (the join of two different atoms is called a line):

(EP) If $l, k_{1}$ and $k_{2}$ are lines such that $l\left\|k_{1}, l\right\| k_{2}$ and if $k_{1} \wedge k_{2} \neq 0$ then $k_{1}=k_{2}$.

THEOREM. Let $L$ be an upper continuous atomistic lattice of length $\geqq 4$. Then, $L$ is a Wilcox lattice if and only if $L$ is weakly modular, M-symmetric and satisfies the axiom (EP), that is, $L$ is an affine matroid lattice.

This theorem follows from Theorem 6.2, by Remark 6.2 and by the results given in [2], pp. 307-309 (Theorem 5 and Prop. 9). Note that our definition of an incomplete element $(\neq 1)$ coinsides with that in [2], p. 307.

REMARK 6.4. About the uniqueness of the modular extension of a Wilcox lattice, we can prove the following results (see [11]). Here the proofs are omitted.

(1) Let $L \equiv \Lambda-S$ be a semicomplemented Wilcox lattice of 
length $\geqq 3$. Then $L$ is modular if and only if $S$ is empty.

(2) Let $L$ be an $\mathscr{C}$-SSC Wilcox lattice of length $\geqq 3$. The modular extension of $L$ is uniquely determined up to isomorphism.

We remark that any $\mathscr{C}-S S C$ Wilcox lattice of length 2 has exactly two modular extensions and that a Wilcox lattice of length $\geqq 3$ may have two modular extensions if it is not $\mathscr{C}-S S C$.

\section{REFERENCES}

1. G. Birkhoff, Lattice theory, third edition, New York, 1967.

2. M. L. Dubreil-Jacotin, L. Leisieur and R. Croisot, Leçons sur la théorie des treillis des structures algébriques ordonnées et des treillis géométriques, Paris, 1953.

3. M. F. Janowitz, Section semicomplemented lattices, Math. Z., 108 (1968), 63-76.

4. F. Maeda, Modular centers of affine matroid lattices, J. Sci. Hiroshima Univ., Ser. A-I 27 (1963), 73-84.

5. - Parallel mappings and comparability theorems in affine matroid lattices, ibid. 27 (1963), 85-96.

6. - Point-free parallelism in Wilcox lattices, ibid. 28 (1964), 10-32.

7. S. Maeda, On atomistic lattices with the covering property, ibid. 31 (1967), 105-121.

8. D. Sachs, Partition and modulated lattices, Pacific J. Math., 11 (1961), 325-345.

9. L. R. Wilcox. Modularity in the theory of lattices, Ann. of Math., 40 (1939), 490505.

10. - A note on complementation in lattices, Bull. Amer. Math. Soc., 48 (1942), 453-458.

11. F. Maeda and S. Maeda, Theory of symmetric lattices, Berlin, 1970.

Received May 25, 1970 and in revised form March 3, 1971.

Ehime UNIVERsity

MAtsuyama, JaPAN 



\title{
PACIFIC JOURNAL OF MATHEMATICS
}

\author{
EDITORS
}

\author{
H. SAMelson \\ Stanford University \\ Stanford, California 94305 \\ C. R. HoBBy \\ University of Washington \\ Seattle, Washington 98105
}

J. DugunduI

Department of Mathematics

University of Southern California

Los Angeles, California 90007

RICHARD ARENS

University of California

Los Angeles, California 90024

\section{ASSOCIATE EDITORS}

\author{
E. F. BeCKENBACH
}

B. H. NEUMANN

F. WoLF

K. YOSHIDA

\section{SUPPORTING INSTITUTIONS}

\author{
UNIVERSITY OF BRITISH COLUMBIA \\ CALIFORNIA INSTITUTE OF TECHNOLOGY \\ UNIVERSITY OF CALIFORNIA \\ MONTANA STATE UNIVERSITY \\ UNIVERSITY OF NEVADA \\ NEW MEXICO STATE UNIVERSITY \\ OREGON STATE UNIVERSITY \\ UNIVERSITY OF OREGON \\ OSAKA UNIVERSITY \\ UNIVERSITY OF SOUTHERN CALIFORNIA
}

\author{
STANFORD UNIVERSITY \\ UNIVERSITY OF TOKYO \\ UNIVERSITY OF UTAH \\ WASHINGTON STATE UNIVERSITY \\ UNIVERSITY OF WASHINGTON \\ AMERICAN MATHEMATICAL SOCIETY \\ CHEVRON RESEARCH CORPORATION \\ NAVAL WEAPONS CENTER
}

The Supporting Institutions listed above contribute to the cost of publication of this Journal, but they are not owners or publishers and have no responsibility for its content or policies.

Mathematical papers intended for publication in the Pacific Journal of Mathematics should be in typed form or offset-reproduced, (not dittoed), double spaced with large margins. Underline Greek letters in red, German in green, and script in blue. The first paragraph or two must be capable of being used separately as a synopsis of the entire paper. The editorial "we" must not be used in the synopsis, and items of the bibliography should not be cited there unless absolutely necessary, in which case they must be identified by author and Journal, rather than by item number. Manuscripts, in duplicate if possible, may be sent to any one of the four editors. Please classify according to the scheme of Math. Rev. Index to Vol. 39. All other communications to the editors should be addressed to the managing editor, Richard Arens, University of California, Los Angeles, California, 90024.

50 reprints are provided free for each article; additional copies may be obtained at cost in multiples of 50 .

The Pacific Journal of Mathematics is published monthly. Effective with Volume 16 the price per volume (3 numbers) is $\$ 8.00$; single issues, $\$ 3.00$. Special price for current issues to individual faculty members of supporting institutions and to individual members of the American Mathematical Society: $\$ 4.00$ per volume; single issues $\$ 1.50$. Back numbers are available.

Subscriptions, orders for back numbers, and changes of address should be sent to Pacific Journal of Mathematics, 103 Highland Boulevard, Berkeley, California, 94708.

PUBLISHED BY PACIFIC JOURNAL OF MATHEMATICS, A NON-PROFIT CORPORATION

Printed at Kokusai Bunken Insatsusha (International Academic Printing Co., Ltd.), 7-17, Fujimi 2-chome, Chiyoda-ku, Tokyo, Japan. 


\section{Pacific Journal of Mathematics}

\section{Vol. 37, No. $3 \quad$ March, 1971}

Mohammad Shafqat Ali and Marvin David Marcus, On the degree of the

minimal polynomial of a commutator operator ................ 561

Howard Anton and William J. Pervin, Integration on topological

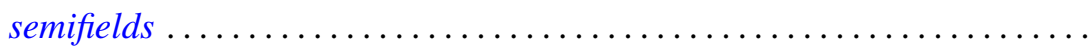

Martin Bartelt, Multipliers and operator algebras on bounded analytic

functions .................................... 575

Donald Earl Bennett, Aposyndetic properties of unicoherent continua ...... 585

James W. Bond, Lie algebras of genus one and genus two ............. 591

Mario Borelli, The cohomology of divisorial varieties ............... 617

Carlos R. Borges, How to recognize homeomorphisms and isometries ....... 625

J. C. Breckenridge, Burkill-Cesari integrals of quasi additive interval

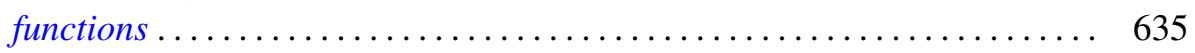

J. Csima, A class of counterexamples on permanents ................ 655

Carl Hanson Fitzgerald, Conformal mappings onto $\omega$-swirly domains . . . . . . 657

Newcomb Greenleaf, Analytic sheaves on Klein surfaces .............. 671

G. Goss and Giovanni Viglino, C-compact and functionally compact

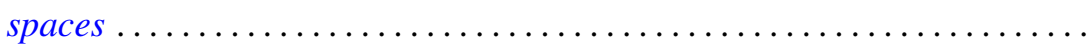

Charles Lemuel Hagopian, Arcwise connectivity of semi-aposyndetic plane

continua ..................................... 683

John Harris and Olga Higgins, Prime generators with parabolic limits ...

David Michael Henry, Stratifiable spaces, semi-stratifiable spaces, and their

relation through mappings .......................

Raymond D. Holmes, On contractive semigroups of mappings ........... 701

Joseph Edmund Kist and P. H. Maserick, BV-functions on semilattices ....... 711

Shûichirô Maeda, On point-free parallelism and Wilcox lattices ........... 725

Gary L. Musser, Linear semiprime $(p ; q)$ radicals ................. 749

William Charles Nemitz and Thomas Paul Whaley, Varieties of implicative

semilattices..................................... 759

Jaroslav Nešetřil, A congruence theorem for asymmetric trees ............ 771

Robert Anthony Nowlan, A study of $H$-spaces via left translations .......... 779

Gert Kjærgaard Pedersen, Atomic and diffuse functionals on a $C^{*}$-algebra ... 795

Tilak Raj Prabhakar, On the other set of the biorthogonal polynomials

suggested by the Laguerre polynomials...

801

Leland Edward Rogers, Mutually aposyndetic products of chainable

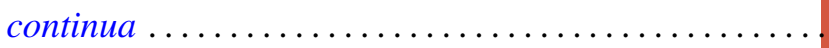

Frederick Stern, An estimate for Wiener integrals connected with squared

error in a Fourier series approximation.

Leonard Paul Sternbach, On k-shrinking and k-boundedly complete basic

sequences and quasi-reflexive spaces .................... 817

Pak-Ken Wong, Modular annihilator $A^{*}$-algebras ........ 